\title{
L'État, la « houle blanche » et le grand capital. L'aliénation des ressources hydrauliques du domaines public québécois au début du XX ${ }^{\mathbf{e}}$ siècle
}

\section{Claude Bellavance}

Volume 51, numéro 4, printemps 1998

URI : https://id.erudit.org/iderudit/005328ar

DOI : https://doi.org/10.7202/005328ar

Aller au sommaire du numéro

Éditeur(s)

Institut d'histoire de l'Amérique française

ISSN

0035-2357 (imprimé)

1492-1383 (numérique)

Découvrir la revue

Citer cet article

Bellavance, C. (1998). L’État, la « houle blanche » et le grand capital. L'aliénation des ressources hydrauliques du domaines public québécois au début du XX ${ }^{\mathrm{e}}$ siècle. Revue d'histoire de l'Amérique française, 51(4), 487-520. https://doi.org/10.7202/005328ar

\section{Résumé de l'article}

Nous livrons ici les résultats d'une étude portant sur la gestion des ressources hydrauliques du domaine public québécois depuis la fin du XIXe siècle jusqu'aux années 1940, période déterminante pendant laquelle furent concédées la majeure partie des ressources hydroélectriques du Québec méridional. Il ressort notamment de l'enquête que le laissez- faire qui caractérise la politique de l'État québécois jusque vers 1910, combiné à l'insuffisance des données alors disponibles, auraient tout d'abord entraîné un développement déséquilibré des potentiels énergétiques. La réforme du régime d'aliénation de la ressource hydrique, puis la création d'un organisme gouvernemental chargé d'en dresser l'inventaire, représentent autant de tentatives pour corriger la situation. L'étude conclut que l'impact négatif du laxisme inital de l'État devait se répercuter sur plusieurs décennies et que la montée en force des réseaux régionaux, au cours des années 1920, a favorisé la rationalisation des conditions d'exploitation des potentiels, tout en consacrant l'appropriation de la res- source par des intérêts privés, de même que le caractère désormais hautement monopoliste de l'industrie de l'électricité.
Tous droits réservés (C Institut d'histoire de l'Amérique française, 1998
Ce document est protégé par la loi sur le droit d'auteur. L’utilisation des services d’Érudit (y compris la reproduction) est assujettie à sa politique d'utilisation que vous pouvez consulter en ligne. 


\title{
L'ÉTAT, LA «HOUILLE BLANCHE» ET LE GRAND CAPITAL. L'ALIÉNATION DES RESSOURCES HYDRAULIQUES DU DOMAINE PUBLIC QUÉBÉCOIS AU DÉBUT DU XX ${ }^{\mathrm{e}}$ SIÈCLE ${ }^{1}$
}

\author{
CLAUDE BELLAVANCE \\ Centre interuniversitaire d'études québécoises \\ Université du Québec à Trois-Rivières
}

\begin{abstract}
RÉSUMÉ
Nous livrons ici les résultats d'une étude portant sur la gestion des ressources hydrauliques du domaine public québécois depuis la fin du XIX ${ }^{\mathrm{e}}$ siècle jusqu'aux années 1940, période déterminante pendant laquelle furent concédées la majeure partie des ressources hydroélectriques du Québec méridional. Il ressort notamment de l'enquête que le laissezfaire qui caractérise la politique de l'État québécois jusque vers 1910, combiné à l'insuffisance des données alors disponibles, auraient tout d'abord entraîné un développement déséquilibré des potentiels énergétiques. La réforme du régime d'aliénation de la ressource hydrique, puis la création d'un organisme gouvernemental chargé d'en dresser l'inventaire, représentent autant de tentatives pour corriger la situation. L'étude conclut que l'impact négatif du laxisme inital de l'État devait se répercuter sur plusieurs décennies et que la montée en force des réseaux régionaux, au cours des années 1920, a favorisé la rationalisation des conditions d'exploitation des potentiels, tout en consacrant l'appropriation de la ressource par des intérêts privés, de même que le caractère désormais hautement monopoliste de l'industrie de l'électricité.
\end{abstract}

\begin{abstract}
This paper deals with the State's management of Quebec's hydraulic resources from the end of the $19^{\text {th }}$ century until the 1940s, a crucial period during which most of the hydraulic resources of the southern part of the Province were conceded. The study shows that the State's policy of inaction up to 1910, combined with the inadequacy of available information, have caused an unbalanced development of the energetic potential. The reform of the public water power policy, and then the creation of a governmental agency responsible for drawing up the hydraulic resource inventory, may be seen as different ways to deal

1. Cette recherche a bénéficié de l'appui financier du Conseil de recherches en sciences humaines du Canada, du Fonds FCAR et du Fonds institutionnel de recherche de l'Université du Québec à TroisRivières. Nous tenons à remercier Roger Levasseur, France Normand et Normand Séguin ainsi que les évaluateurs anonymes de la revue pour leurs commentaires. Nous voulons également souligner la contribution de nos assistants de recherche, Samuel Blouet, Daniel Boutet, François Charest et Marie-Josée Dorion.
\end{abstract}

RHAF, vol. 51, ${ }^{\circ}$ 4, printemps 1998 
with the problem. The study also comes to the conclusion that the negative impact of the State's initial «laissez-faire» had consequential effects over many decades, and that the building of regional networks during the 1920s contributed to the rationalization of production and sanctioned the appropriation of the resource by private interests as well as the henceforth highly monopolistic nature of the electrical industry.

Depuis la fin du siècle dernier, l'hydroélectricité a été au cœur de la transformation de l'économie québécoise. Le Québec, c'est bien connu, recèle d'importants potentiels hydrauliques et l'aménagement des sites inexploités représente aujourd'hui comme hier un sujet d'actualité. Or, l'histoire des modes d'appropriation et de la mise en valeur de cette ressource naturelle demeure un domaine d'étude peu exploré. Certains travaux consacrés à l'industrie de l'électricité ont traité — généralement dans une perspective d'histoire des affaires - des projets d'aménagement de centrales ${ }^{2}$. Aucune étude, cependant, n'a encore tenté de rendre compte plus largement de la politique de l'État québécois en matière de développement hydroélectrique. Le présent article constitue une première étape dans ce sens ${ }^{3}$. Il propose une vue d'ensemble des modes d'aliénation des ressources hydrauliques du domaine public depuis la fin du XIX ${ }^{\mathrm{e}}$ siècle jusqu'aux années 1940 tout en tentant d'évaluer leur impact. Plus particulièrement, il s'attache à mettre en évidence les nouveaux impératifs de rationalisation de l'exploitation des cours d'eau qui se font sentir dès les premières décennies du siècle.

2. Voir, entre autres, Clarence Hogue, André Bolduc et Daniel Larouche, Québec, un siècle d'électricité (Montréal, Libre Expression, 1979), 381 p.; David P. Massell, Amassing Power in a Northern Landscape: J. B. Duke and the Development of the Saguenay River, 1897-1927, thèse de Ph.D., Duke University, 1997, 451 p.; Claude Bellavance, Shawinigan Water and Power, 1898-1963. Formation et déclin d'un groupe industriel au Québec (Montréal, Boréal, 1994), 448 p. La question de l'appropriation des ressources du domaine public québécois est abordée brièvement dans C. Armstrong et $\mathrm{H}$. V. Nelles, Monopoly's Moment. The Organization and Regulation of Canadian Utilities, 1830-1930 (Toronto, University of Toronto Press, 1988), 393 p., de même que dans J. H. Dales, Hydroelectricity and Industrial Development in Quebec, 1898-1940 (Cambridge [Mass.], Harvard University Press, 1957), 269 p.

3. Depuis quelques années, nous menons un projet de recherche qui vise à appréhender, dans une démarche unifiée, les multiples aspects du développement du secteur de l'électricité au Québec: impact sur l'activité économique, stratégies des entreprises au regard des régulations marchandes et des visées de l'État, diffusion des usages de l'électricité sous l'angle des pratiques de consommation et de la transformation des modes d'existence, émergence de nouvelles représentations du rapport à la nature etc. Voir Claude Bellavance, Shawinigan Water and Power, 1898-1963, op. cit.; «Réseaux, territoires et électricité: la dynamique spatiale du processus d'électrification du Québec méridional», dans Serge Courville et Normand Séguin, dir., Espace et culture/Space and Culture (Sainte-Foy, Les Presses de l'Université Laval, 1995), 393-404; «Un long mouvement d'appropriation: de la première à la seconde nationalisation», dans Yves Bélanger et Robert Comeau, dir., Hydro-Québec, autres temps, autres défis (Québec, Presses de l'Université du Québec, 1995), 71-78; «La puissance de l'eau», dans Claude Boudreault, Serge Courville et Normand Séguin, dir., Atlas historique du Québec. Le territoire (Sainte-Foy, Les Presses de l'Université Laval, 1997), 85-93. 
Nous avons choisi d'orienter nos investigations dans trois grandes directions. La première renvoie à la reconnaissance et à la mise en valeur de la ressource ${ }^{4}$. La «houille blanche» - comme la comparaison implicite avec le charbon le suggère - fut très tôt considérée comme un moyen de favoriser le développement industriel du Québec. À la fin des années 1890, la plupart des sites hydrauliques convoités par les entrepreneurs privés relevaient encore du domaine public. Leur aménagement était alors conditionnel à leur aliénation par l'État suivant deux modes distincts: la vente en pleine propriété ou le bail à long terme. À cette époque, on n'envisageait pas encore la possibilité qu'un organisme gouvernemental ou une société de la Couronne assume la construction et l'exploitation d'ouvrages hydrauliques ou hydroélectriques. Dans ces années pionnières, les sites les plus prometteurs paraissaient souvent hors d'atteinte, parce qu'éloignés des centres de consommation et des principales voies de communication. En fait, les pouvoirs publics n'avaient qu'une connaissance approximative de la puissance de ces chutes et rapides, disséminés au surplus sur un vaste territoire. Quelle fut l'ampleur des potentiels aliénés? Qui devait s'en porter acquéreur, et dans quelles conditions? La deuxième direction empruntée prend en considération l'émergence d'un nouveau système technique. Au tournant du siècle, la puissance des cours d'eau était encore souvent consommée sur place, sous la forme d'énergie mécanique. Mais le perfectionnement des techniques de captation, de transformation et de transport de l'énergie annonçait déjà la montée en force de la filière hydroélectrique. Comment et à quel moment l'État québécois a$\mathrm{t}$-il pris acte de ces progrès? Enfin, la troisième direction de recherche nous conduit à analyser les conséquences de la monopolisation de l'industrie de l'électricité. Par l'ampleur des capitaux investis et son impact sur la croissance industrielle, cette industrie devint rapidement l'un des secteurs-clés de l'économie québécoise. Tirant profit des économies d'échelles qu'entraînait l'aménagement de grandes centrales hydroélectriques, une poignée d'entreprises d'envergure régionale parvinrent, à compter des années 1910, à éclipser les petites compagnies locales (sociétés privées ou services municipalisés) qui, jusque-là, assuraient l'approvisionnement en électricité. Dans quelle mesure le grand capital a-t-il pu infléchir la politique gouvernementale en matière de développement des ressources hydroélectriques?

Cette étude pose plus largement le problème de la transformation des régulations étatiques en contexte de développement du capitalisme de

4. La reconnaissance de la ressource a constitué une première étape dans le processus d'appropriation d'un territoire immense: Claude Bellavance, «La puissance de l'eau», op. cit. Pour une approche relationnelle de la notion de ressources, voir Claude Raffestin, Pour une géographie du pouvoir (Paris, Litec, 1980), 203-213. 
monopole. Il s'agit d'un sujet de recherche très vaste et encore peu

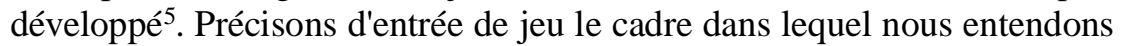
situer notre analyse. Nous voulons d'abord éviter toute conception par trop instrumentale du rôle de l'État; pensons, par exemple, aux analyses fonctionnalistes ou à celles qui n'ont reconnu en lui que l'expression du pouvoir de la classe dominante. De même récusons-nous les approches que l'on pourrait qualifier de «substantialistes», qui chercheraient à définir l'État en lui-même, et conduiraient à une représentation idéalisée, en faisant même une force quasi intemporelle, au-dessus de la société ${ }^{6}$. Les développements des dernières décennies en sciences sociales - par-delà les écoles et les disciplines considérées - nous invitent au contraire à appréhender celui-ci comme un rapport social à part entière, lequel se transforme dans le temps et prend des formes variables selon les sociétés observées; bref, à adopter un mode d'approche relationnel, fondé sur l'analyse du changement structurel.

S'inscrivant dans le courant régulationniste ${ }^{7}$, l'économiste Robert Delorme suggère ainsi de distinguer quatre places (ou dimensions,

5. On consultera notamment le chapitre intitulé «The Utility of Regulation» dans C. Armstrong et H. V. Nelles, Monopoly's Moment, op. cit., 248-269. Quelques travaux sur le Québec ou le Canada ont paru depuis quelques années qui réfèrent à l'approche régulationniste dont il sera rapidement question dans les lignes qui suivent. Voir, notamment, G. Boismenu et D. Drache, Politique et régulation: modèle de développement et trajectoire canadienne (Montréal, Éditions du Méridien, 1990), 360 p.; de même que J. Létourneau, Croissance économique et régulation duplessiste: retour sur les origines de la Révolution tranquille (Québec, Université Laval, 1986), 62 p.

6. Hegel, qui voyait dans l'État moderne l'avènement du sujet universel, est certes celui qui a été le plus loin dans cette direction. Il existe une littérature abondante sur ce sujet depuis Marx jusqu'aux politologues ou sociologues contemporains. On pourra consulter Henri Lefebvre, De l'État (Paris, Union générale d'éditions, 1976), 2: 118-164, Raymond Boudon et Francois Bourricaud, dir., Dictionnaire critique de la sociologie (Paris, Presses universitaires de France, 1990), 232-242 et Alain Touraine, Critique de la modernité (Paris, Fayard, 1992), 102-106.

7. Cette école, née en France durant les années 1970, connaît aujourd'hui un certain rayonnement. Les régulationnistes ont tenté notamment de mieux intégrer la dimension «institutionnelle» dans l'analyse économique. Ils insistent en outre sur les rapports complexes et changeants qu'entretient l'État avec le marché, voire avec l'économie en général. Michel Aglietta, Régulation et crises du capitalisme: l'expérience des États-Unis (Paris, Calmann-Levy, 1976), 334 p.; Robert Boyer, La théorie de la régulation: une analyse critique (Paris, La Découverte, 1986), 143 p.; Robert Boyer et Yves Saillard, dir., Théorie de la régulation: l'état des savoirs (Paris, La Découverte, 1995), 568 p. Le courant des institutionnalistes américains tentent aussi de prendre en considération des dimensions de la vie sociale que le modèle dominant rejette comme extérieur à l'analyse économique. Ces approches conservent cependant les paradigmes de l'économie néo-classique (individualisme méthodologique, rationalité, équilibre etc.). Douglass C. North, Institutions, Institutional Change and Economic Performance (New York, Cambridge University Press, 1990), 160 p. Pour un aperçu des différences et des convergences entre ces grands courants: Marie-Claire Villeval, «Une théorie économique des institutions?», dans Robert Boyer et Yves Saillard, dir., Théorie de la régulation, op. cit., 479-489. Sur la question des nouveaux rapports au marché, voir Michel Hollard, «Les formes de la concurrence», dans Robert Boyer et Yves Saillard, dir., Théorie de la régulation, op. cit., 162-170. 
dirons-nous) pour rendre compte des modes de relation entre l'État et l'économie ${ }^{8}$ : 1 - État comme principe sociétal (ce qui renvoie à la distinction entre société civile et État); 2 - État comme support des formes institutionnelles garantes des règles du jeu de la vie en société dans un contexte historique donné ${ }^{9} ; 3$ - État comme hiérarchie publique (on pourrait dire comme organisation ou comme bureaucratie, au sens qu'accordait Max Weber à ce concept); 4 - État comme acteur social. Les deuxième et quatrième de ces dimensions nous interpelleront plus spécifiquement, l'analyse des fondements du pouvoir de l'État contemporain de même que l'étude de sa dynamique interne (ou du développement de la fonction publique) débordant évidemment le cadre du présent article. Appréhendé à la fois comme structure et comme acteur, l'État tiendra en fait ici trois rôles essentiels: un rôle de contrainte, un rôle de légitimation et un rôle de coordination.

Ces rôles ont été assumés de diverses manières selon les périodes considérées. Ainsi, tout au long du XXe siècle, l'État a considérablement étendu son influence dans le champ économique. De son affirmation en tant que coordinateur du système productif au cours de la Première Guerre mondiale à l'institutionnalisation des diverses formes de prestations sociales et à l'élargissement des prélèvements fiscaux, en passant par la création de banques centrales et l'établissement de premières véritables politiques de développement, une profonde transformation de la relation entre l'État et l'économie se profile. Pour qualifier cette mutation, les chercheurs ont utilisé des notions comme «État keynésien», «État providence», «État interventionniste», «État inséré» qu'ils ont opposé à «Laissez-faire», «État libéral», «État veilleur de nuit», «État circonscrit» etc. On s'accorde généralement pour voir dans les années 1930 et 1940 une période charnière qui mar-

8. Voir «L'état relationnel intégré complexe», dans Robert Boyer et Yves Saillard, dir., Théorie de la régulation, op. cit., 180-188. Le sociologue Alain Touraine propose un mode d'approche qui n'est pas sans rapport avec le projet des régulationnistes. Voir notamment Production de la société (Paris, Seuil, 1993), 220-241 et La voix et le regard (Paris, Seuil, 1978), 136144, de même que Paul R. Bélanger, et Benoît Lévesque, «La "théorie" de la régulation, du rapport salarial au rapport de consommation. Un point de vue sociologique», Cahiers de recherche sociologique, 17 (1991): 17-51.

9. Ce sont l'élaboration du droit et son contrôle par la justice et la police, la reconnaissance et la codification des autres institutions, les règles de fonctionnement des pouvoirs législatifs et exécutifs, les règles de financement et de prélèvement, les règles de relations avec les autres États. Ces formes institutionnelles se présentent comme des compromis institutionnalisés, c'est-à-dire une série de codifications qui fixent, en quelque sorte, les règles du jeu en société. Voir aussi, du même auteur: «État et hétérogénéité: ERIC et le MPPE», Cahiers de recherche sociologique, 17 (1991): 153-183. 
que définitivement le passage aux nouveaux rapports État/économie et leur intégration dans les représentations du monde social ${ }^{10}$.

Dans le domaine de l'électricité au Québec, cette transformation des rapports de l'État à l'économie culminera au début des années 1960, avec la prise en charge de l'ensemble du secteur par une entreprise publique. Aussi importante qu'ait été cette mesure en regard du contexte politique et social de cette époque, la seconde étatisation peut être vue aussi comme l'aboutissement d'un long processus que nous avons interprété ailleurs comme la réappropriation d'un espace d'intervention dans le champ économique ${ }^{11}$. Pareil mouvement n'a toutefois rien de linéaire. Les recherches montrent plutôt que les organismes de réglementation de l'industrie de l'électricité ont souvent été incapables de réaliser l'intégralité de leur mandat initial. Ce fut le cas, par exemple, de la Commission des services publics, dont les pouvoirs contraignants furent remis en cause par les grands distributeurs peu après sa création, en $1909^{12}$. La régie publique qui l'a remplacée au milieu des années 1930 fut dotée de pouvoirs plus étendus et a eu une influence considérable, en fixant les tarifs, notamment. Toutefois, elle n'a pas été non plus à l'abri ni des pressions politiques ni de celles des entreprises d'électricité, comme en témoignent les changements de direction et les réformes de sa loi constituante qui ont accompagné chaque changement de gouvernement à Québec entre 1935 et 1960, de même que sa «discrétion» au cours de la décennie précédant la seconde nationalisation ${ }^{13}$. Nous verrons plus loin qu'une telle succession d'avancées et de reculs a également caractérisé l'intervention de l'État en matière de gestion des ressources hydroélectriques. Celui-ci n'en fut pas moins un agent de régulation indispensable pour le développement de l'industrie de l'électricité, exerçant même de manière parfois inédite ses rôles de coordination, de légitimation et de contrôle.

10. Non seulement assiste-t-on alors au déploiement des appareils gouvernementaux et à l'extension du champ de leurs compétences, mais c'est à ce moment que les économistes hétérodoxes (Beveridge, Keynes et leurs disciples) apportent leur caution aux politiques gouvernementales interventionnistes formulées d'abord dans le but de surmonter la crise et ensuite pour coordonner l'effort de guerre.

11. Claude Bellavance, Shawinigan Water and Power, 1898-1963, op. cit., 307-309; «Un long mouvement d'appropriation. De la première à la seconde nationalisation», dans Yves Bélanger et Robert Comeau, Hydro-Québec, autres temps, autres défis, op. cit., 71-78.

12. C. Armstrong et H. V. Nelles, Monopoly's Moment, op. cit., 203-206 et Claude Bellavance et Paul-André Linteau, «La diffusion de l'électricité à Montréal au début du $\mathrm{XX}^{\mathrm{e}}$ siècle», dans Horacio Capel et Paul-André Linteau, dir., Barcelona-Montréal. Développement urbain comparé (Barcelone, Éditions de la Universidad de Barcelona) (à paraître).

13. Claude Bellavance, Shawinigan Water and Power, 1898-1963, op. cit., 159-168. 
Pour mener à bien la présente recherche, nous avons consulté les rapports annuels de la Commission des eaux courantes de même que ceux du service hydraulique du ministère des Terres et Forêts. Les inventaires des sites concédés par l'État publiés dans les Documents de la Session du Québec entre 1900 et 1947 ont fait l'objet d'une exploitation systématique ${ }^{14}$. Nous avons par ailleurs procédé à l'analyse de la législation québécoise relative à l'électricité depuis les années 1880 jusqu'à 1950. D'autres sources, comme la brochure Central Electric Stations, une publication du gouvernement fédéral qui, à quatre occasions entre 1918 et 1928, dresse un portrait détaillé des aménagements hydroélectriques et des marchés de l'électricité pour chaque municipalité du Québec, ont également été mises à profit. Enfin, nous avons eu recours aux fonds de compagnies privées conservés aux Archives d'Hydro-Québec.

\section{A- LE CADRE INITIAL DE L'ALIÉNATION DES RESSOURCES HYDRAULIQUES DU DOMAINE PUBLIC}

L'exploitation de l'énergie hydraulique est une réalité ancienne. Si les hommes ont rapidement appris à modifier le cours des rivières pour assurer l'approvisionnement en eau des populations et pour irriguer les terres, et que des capteurs de la force hydraulique furent conçus dès l'Antiquité, il faut attendre l'époque médiévale avant que les moulins à eau ne viennent concurrencer la traction animale et la force humaine dans le bilan énergétique des sociétés ${ }^{15}$. La roue hydraulique allait jouer un rôle primordial jusqu'à l'essor de la machine à vapeur à la fin du XVIII ${ }^{\mathrm{e}}$ siècle. Au Québec comme en Nouvelle-Angleterre, des ouvrages ont été érigés en bordure des voies d'eau dès l'époque coloniale; leur

14. Les informations contenues dans ces inventaires ont été saisies et traitées à l'aide d'une base de données relationnelle (472 mentions de cessions de sites et 2914 évaluations de potentiels). Une première étape de jumelage des sources a permis de constituer des séries temporelles et de comparer la puissance nominale des sites aux fins de l'enregistrement des droits de propriétés (ou, dans le cas des locations, pour établir le montant des redevances) avec les évaluations réalisées périodiquement par les services hydrauliques canadiens et québécois (évaluations faites dans le but de mettre à jour les données disponibles en tenant compte de l'évolution des techniques d'exploitation).

15. Voir en particulier Marc Bloch, «Avènement et conquêtes du moulin à eau», Annales d'histoire économique et sociale, 36 (1935): 538-563; Jean-Claude Debeir, Jean-Paul Deléage et Daniel Hémery, Les servitudes de la puissance: une histoire de l'énergie (Paris, Flammarion, 1986), 117-173; Bertrand Gille, Histoire des techniques: technique et civilisations, technique et sciences (Paris, Gallimard, 1978), 603-664; A. P. Usher, A History of Mechanical Invention [1929] (New York, Dover, 1982), 116-186; Peter Temin, «Steam and Waterpower in the Early 19th Century», dans Robert W. Fogel et Stanley L. Engerman, dir., The Reinterpretation of America Economic History (New York, Harper \& Row, 1971), 228-237; Robert B. Gordon, «Hydrological Science and the Development of Waterpower for Manufacturing», Technology and Culture, 26,2 (1985): 204-235. 
nombre est allé grandissant tout au long du XIX ${ }^{\mathrm{e}}$ siècle $^{16}$. À cette époque, les dispositifs pour exploiter la puissance de l'eau étaient encore modestes. L'émergence de l'hydroélectricité devait bientôt lancer sur des bases nouvelles la mise en valeur des ressources énergétiques des rivières. Initialement produite à partir du charbon, l'électricité trouvait en la force hydraulique une source d'énergie primaire à très bon marché qui allait progressivement lui permettre de remplacer la machine à vapeur au sein du système productif. Au même moment, l'énergie électrique commença à se répandre dans d'autres sphères des activités humaines: transport public, éclairage des rues et des résidences, moyens de communication hertzienne, électrochimie et électrométallurgie etc. L'électrification - ce mouvement de fond qui touche les pays industrialisés durant le premier tiers du $\mathrm{XX}^{\mathrm{e}}$ siècle, et qui vit tout à la fois l'avènement de la production d'électricité à grande échelle, la constitution de grands réseaux de transport et de distribution de même que la transformation des profils de consommation énergétique et des styles de vie des sociétés contemporaines - était dès lors enclenchée.

\section{1- La juridiction du Québec}

À l'instar de bien d'autres sociétés, le Québec veilla très tôt à préserver le caractère public des voies d'eau navigables. Ainsi, dès le Régime français, le lit du fleuve et des rivières navigables devait faire partie intégrante du domaine public, tandis que celui des cours d'eau impropres à la navigation relevait du seigneur, seul habilité à y ériger des moulins. À partir du milieu du XIX ${ }^{\mathrm{e}}$ siècle, la commutation des censives en propriétés privées conférera au lit des cours d'eau non navigables le même statut juridique que les autres types de propriétés foncières ${ }^{17}$, cependant que

16. En 1871, après une première poussée industrielle, les grands équipements tels les moulins à carder et à fouler, les moulins à scie et les moulins à farine étaient disséminés sur le territoire suivant les ressources du milieu. Les planches du premier tome de l'Atlas historique du Québec sont particulièrement éloquentes à ce sujet. Serge Courville, Jean-Claude Robert et Normand Séguin, Le pays laurentien au XIX ${ }^{e}$ siècle. Les morphologies de base (Sainte-Foy, Les Presses de l'Université Laval, 1995), 95. On consultera aussi Claude Bellavance, «La puissance de l'eau», loc. cit.; Solange De Blois, «Les moulins de Terrebonne (1720-1775) ou les hauts et les bas d'une entreprise seigneuriale», Revue d'histoire de l'Amérique française, 51,1 (été 1997): 39-70; Françoise Noël, «Chambly Mills, 1784-1815», Historical Papers/Communications historiques (1985): 102-116; Corinne Beutler, «Les moulins à farine du Séminaire de Saint-Sulpice à Montréal (1658-1840): essai d'analyse économique d'une prérogative du régime seigneurial», Historical Papers/Communications historiques (1983): 184-207 et Louise Dechêne, Le partage des subsistances au Canada sous le régime français (Montréal, Boréal, 1995), 33 et 35.

17. Les berges et le fond des rivières non navigables devinrent la propriété des riverains qui, moyennant certaines conditions, pouvaient en user à leur guise. Statuts refondus de la Province de Québec (SRQ) 1861, chap. 51, «An Act Respecting the Improvement of Water-Courses», SRQ 1888, art. 5535 et 5536 , «Du droit d'exploitation du cours d'eau par les propriétaires riverains». 
l'État conservait sa pleine souveraineté sur les rivières réputées navigables ${ }^{18}$.

Jusqu'aux années 1880, les moulins étaient généralement de taille modeste et leur impact sur l'environnement limité. Par la suite, on vit progressivement apparaître des établissements dotés d'équipements hydrauliques plus imposants, notamment dans les grandes scieries, les fabriques de textile, les papetières et les centrales hydroélectriques. La construction d'une première grande centrale à Niagara, vers 1890, a marqué un tournant. Elle prouvait qu'il était rentable de transformer l'énergie mécanique des cours d'eau en énergie électrique, puis d'en assurer le transport jusqu'au lieu de consommation ${ }^{19}$. À mesure qu'émergeait la nouvelle filière énergétique, les entrepreneurs et les hommes politiques prenaient conscience de l'immense valeur des rivières québécoises. Mais l'exploitation à vaste échelle de la force hydraulique exigeait une révision de la législation relative à la propriété et aux conditions d'exploitation des cours d'eau. Elle supposait aussi que l'on statue sur l'ordre de gouvernement, provincial ou fédéral, qui pourrait disposer de ces ressources. Bien que cette clarification ne viendra qu'à la fin du siècle, la législature québécoise amenda la loi régissant la concession des terres de la Couronne dès $1884^{20}$, afin de préserver le caractère public des berges et du lit des rivières et des lacs. Quant à la question, en suspens depuis plusieurs décennies, de départager les compétences des deux niveaux de gouvernement, elle fut tranchée en 1898 par une décision du Conseil privé à Londres ${ }^{21}$. Dorénavant, la force hydraulique des cours d'eau — qu'ils soient ou non navigables — allait être considérée comme une richesse naturelle; par conséquent, celle-ci tomberait sous la juridiction des provinces.

\section{2- L'époque du «laissez-faire»}

Confirmé dans ses pouvoirs, l'État québécois allait s'empresser d'utiliser ce nouvel outil de développement industriel en confiant à des intérêts privés

18. O. Lefebvre, «Les forces hydrauliques de la Province de Québec», Revue trimestrielle canadienne (juin 1926): 141-151; A. B. Normandin, «La législation et l'administration des eaux», Revue trimestrielle canadienne (juin 1929): 187-198.

19. T. P. Hughes, «Electrification of America: the System Builders», Technology and Culture, 20 (1979): 124-161; Networks of Power: Electrification in Western Society, 1880-1930 (Baltimore, Johns Hopkins University Press, 1983), 474 p.

20. Il s'agit de l'introduction, par la législature québécoise, d'une clause dite «des trois châ̂nes» (1884). À l'origine, cette mesure législative concernait principalement les droits de pêche. $S R Q$ 1888, art. 1375, «Des baux de pêche».

21. Dans une cause devenue célèbre qui opposait l'Ontario et le gouvernement fédéral. Voir à ce sujet I. Biss, «Water Power», dans W. Stewart Wallace, dir., The Encyclopedia of Canada (Toronto, University Associates of Canada, 1937), volume 6. 
le soin d'aménager les sites hydrauliques sur son territoire ${ }^{22}$. L'examen de la documentation officielle jusqu'à la Première Guerre mondiale nous permettra, d'une part, de voir comment les pouvoirs publics se sont dessaisis de ces potentiels et, d'autre part, de caractériser les nouveaux propriétaires.

Au tournant du siècle, la politique de mise en valeur des ressources énergétiques de l'État québécois s'inscrivait en droite ligne avec son approche traditionnelle. Ainsi, pour un montant généralement minime, celui-ci cédait-il les droits d'exploitation de la force hydraulique des cours d'eau comme il l'eut fait de tout autre propriété, n'exigeant comme seule condition de l'acquéreur qu'il procède à l'aménagement des sites dans des délais déterminés. Selon un relevé officiel datant de $1914^{23}$, 112 sites (89 ventes en pleine propriété et 23 baux) avaient déjà été concédés au cours de la phase pionnière de l'industrie de l'électricité. Le potentiel de la ressource nous est connu dans $68 \%$ des $\operatorname{cas}^{24}$ : au total, il se serait élevé à environ 1,5 million de HP. Ces pouvoirs d'eau étaient de taille fort variable. Le plus modeste des sites, localisé sur la rivière à Beauce dans le comté de Champlain, était apte à fournir une puissance de 24 HP. À l'autre extrême, on établissait la puissance de deux sections de la rivière Saguenay à 200000 HP chacune. La figure 1 illustre la répartition des sites en trois grandes catégories. On remarque que huit d'entre eux sont évalués à moins de $300 \mathrm{HP}$; leur rôle apparaît marginal en cette période de forte augmentation de la taille

22. La politique gouvernementale en ce domaine fut fortement critiquée par certains milieux nationalistes. Henri Bourassa affirmait dès 1904: «J'ai déjà suggéré et je suggère de louer nos pouvoirs hydrauliques, nos pouvoirs d'eau par baux emphytéotiques. Je suis convaincu, du moins, je crois sincèrement, que dans un avenir assez rapproché nos gouvernements se serviront eux-mêmes de nos pouvoirs d'eau qui font l'envie des autres pays et dont on ne peut dire encore la valeur, ni quand ils s'épuiseront. [...] Avant de terminer, laissez-moi vous conseiller encore une fois d'être très prudents au sujet de la vente de nos pouvoirs hydrauliques, de ne pas les vendre de main à main, ni à des prix ridicules: c'est une chose que je déplore sincèrement. J'irai encore plus loin: je crois que nous ne devrions pas vendre nos forces hydrauliques, que d'ici à quelques années, l'État développera lui-même l'électricité; je crois que l'État gardera ses forces hydrauliques, et l'État devra en rester maître. Il arrivera peutêtre qu'avant 25 ans, non seulement l'État ne voudra pas louer ses pouvoirs hydrauliques, mais fera luimême l'électricité pour tout le service public, pour les chemins de fer, pour les tramways, pour l'éclairage.» Témoignage à la Commission sur les affaires de la Colonisation, cité dans T.-D. Bouchard, Les réformes qui s'imposent dans la régie de nos ressources hydro-électriques (Saint-Hyacinthe, L'imprimerie Yamaska, 1935), 91-92. Sur Henri Bourassa, on consultera notamment J. Levitt, Henri Bourassa and the Golden Calf: the Social Program of the Nationalists of Quebec (1900-1914) (Ottawa, Éditions de l'Université d'Ottawa, 1969), 178 p., de même que, du même auteur, Henri Bourassa, critique catholique (Ottawa, Société historique du Canada, 1977), 24 p.

23. Ministère des Terres et Forêts, Service hydraulique (1914), Tableau des Forces Hydrauliques concédées par la Province de Québec, du $1^{\text {er }}$ juillet au 31 décembre 1913.

24. Les sites qui ne font pas l'objet d'une évaluation précise de potentiel ne sont pas nécessairement moins importants pour autant. Ainsi, aux côtés de petites cascades cédées à des entrepreneurs locaux, trouvons-nous certaines chutes considérables comme celles de la rivière Péribonka ou les rapides des Grès sur la Saint-Maurice. 
FIGURE 1

DISTRIBUTION DES SITES CONCÉDÉS ENTRE 1896 ET 1913 SELON LE POTENTIEL, LN(HP)

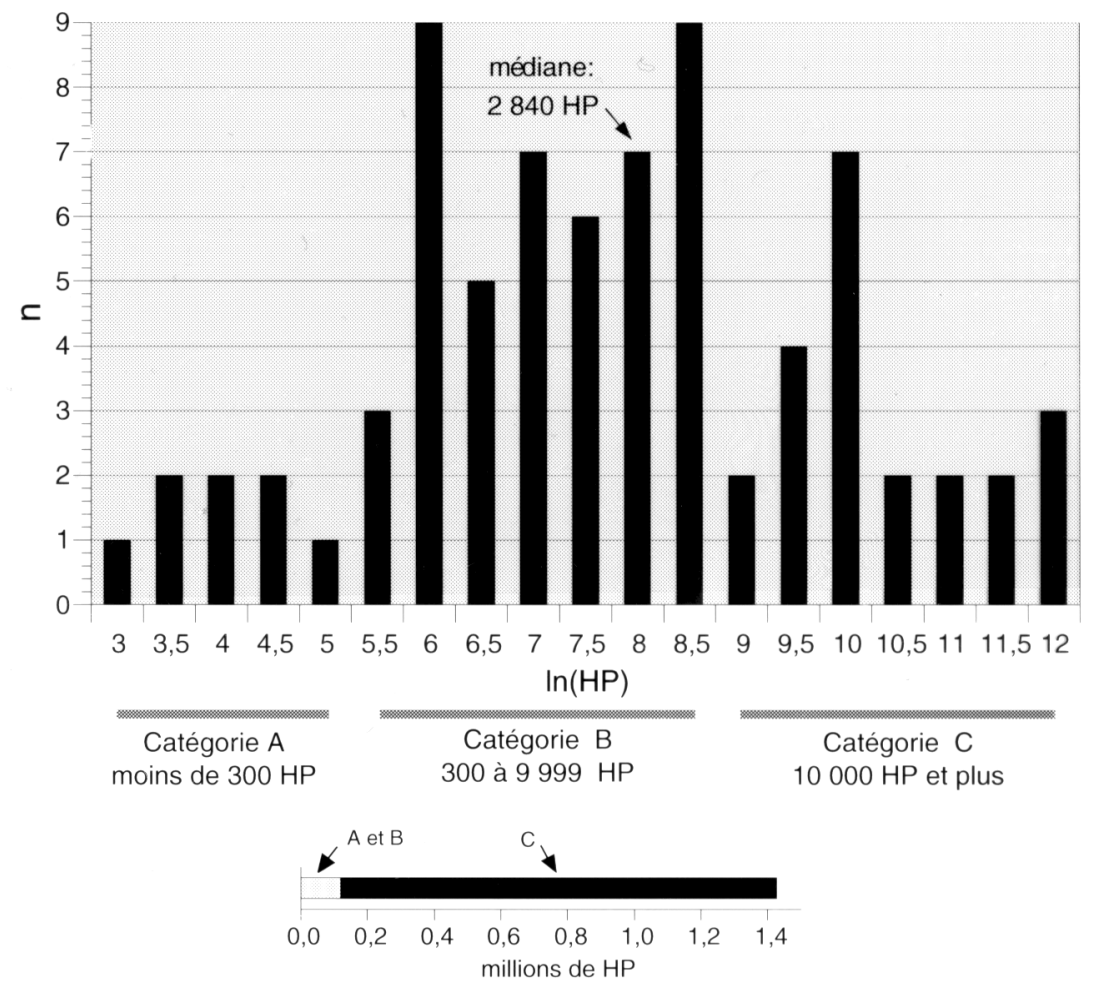

Source: Ministère des Terres et Forêts, Service hydraulique (1914), Tableau des forces hydrauliques concédées par la Province de Québec, du $1^{\mathrm{er}}$ juillet au 31 décembre 1913.

moyenne des dispositifs hydrauliques. Le groupe le mieux représenté (46 unités), la catégorie B, comprend les potentiels se situant entre 300 et 10000 HP. Viennent enfin les très gros potentiels. Ces 22 chutes ou rapides comptent à eux seuls pour plus de $90 \%$ de la force hydraulique cédée par l'État avant 1914. Leur appropriation représentait, on l'aura compris, des enjeux considérables.

On constate, par ailleurs, que les revenus tirés par l'État de l'aliénation des potentiels hydauliques sont très limités. Le prix moyen des 89 sites vendus en pleine propriété avant 1914 est de 3365\$ (écart-type: 8285). Le prix moyen par HP s'établit à près de 1,29\$ (pour les 55 sites qui ont fait l'objet 
FIGURE 2

PRIX DE VENTE DES SITES HYDRAULIQUES SELON LA PUISSANCE ATTRIBUÉE, \$ PAR HP ET LN(HP)

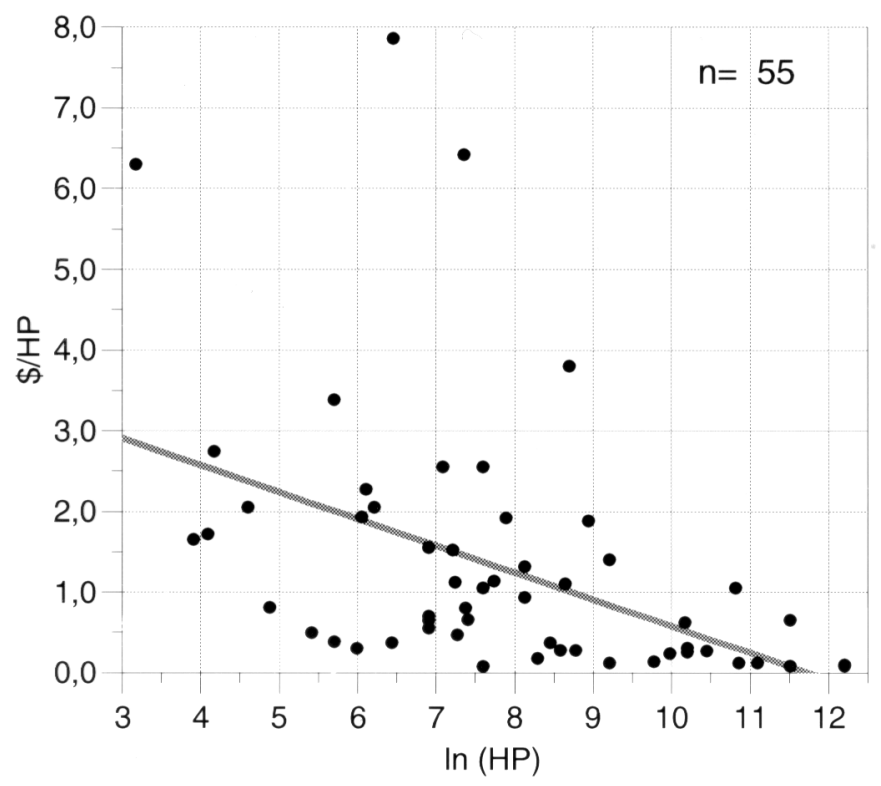

Source: Ministère des Terres et Forêts, Service hydraulique (1914), Tableau des forces hydrauliques concédées par la Province de Québec, du 1er juillet au 31 décembre 1913.

d'une évaluation de potentiel). Ce prix par unité de puissance tend à diminuer sensiblement selon la taille (figure 2): $2,20 \$$ pour les sites de la catégorie $\mathrm{A}, 1,54 \$$ et $0,32 \$$ pour ceux des catégories $\mathrm{B}$ et $\mathrm{C}$. Le montant de base exigé pour les emplacements loués ${ }^{25}$ durant la même période s'élève en moyenne à $15,7 \phi / \mathrm{HP}$ (respectivement $39,5 \phi / \mathrm{HP}, 19,1 \phi / \mathrm{HP}$ et $5,9 \phi / \mathrm{HP}$ pour les catégories $\mathrm{A}, \mathrm{B}$ et $\mathrm{C}$ ).

À ces différentes catégories de sites correspondent divers types de concessionnaires $^{26}$. On retouve en premier lieu les petits exploitants -

25. Nous connaissons la somme exigée des locataires dans 21 des 23 cas.

26. Si l'on s'en tient uniquement aux relevés officiels, 65 des 112 concessionnaires seraient des «individus». Le jumelage avec d'autres sources indique toutefois que plusieurs de ces entrepreneurs étaient en réalité des représentants d'entreprises industrielles ou de compagnies d'électricité. Les données disponibles ne nous permettent malheureusement pas de vérifier l'étendue d'une telle pratique. Il appert néanmoins qu'elle était plus courante avant 1914 et qu'elle avait principalement cours parmi les sites de la catégorie $\mathrm{C}$. 
souvent propriétaires de moulins à scie — qui tirent des cours aménagés quelques dizaines ou, tout au plus, quelques centaines de HP. Un deuxième groupe est formé d'entreprises industrielles qui se sont approprié un potentiel énergétique nettement plus élevé. Il s'agit notamment de compagnies forestières produisant de gros volumes de bois scié et de papetières, dont le nombre augmente rapidement depuis les années 1880. Tout comme les petits exploitants, ces entreprises utilisent directement la force hydraulique des cours d'eau sans la transformer en énergie électrique. Arrivent ensuite les compagnies d'électricité locales et les services municipalisés ${ }^{27}$. On compte également quelques entreprises d'électricité d'envergure régionale en voie de se constituer en grands réseaux (Royal Electric/Montreal Light Heat and Power à Montréal, Shawinigan Water and Power en Mauricie, par exemple) et qui cherchent à profiter des économies réalisées par la production à vaste échelle. Finalement, apparaît le groupe des spéculateurs ou des promoteurs dont les projets quelquefois trop ambitieux ne se sont jamais concrétisés.

En règle générale, le concessionnaire obtient de l'État une portion seulement des forces hydrauliques et du lit d'une rivière. Si les propriétés riveraines nécessaires à l'aménagement du barrage appartiennent encore à la Couronne, elles seront normalement vendues elles aussi. Ces lots, cependant, sont souvent déjà entre les mains d'intérêts privés; le cas échéant, les promoteurs du projet devront donc en négocier l'acquisition au prix fort.

Cette multiplicité des intervenants devait, en pratique, poser toutes sortes de problèmes de gestion des cours d'eau. Concrètement, elle s'est même avérée une entrave à l'aménagement de grandes centrales hydroélectriques. À la faveur des progrès rapides des connaissances dans les domaines de l'hydrographie et de l'ingénierie, la voie était maintenant ouverte à l'érection d'ouvrages de plus en plus puissants et efficaces. Ainsi, pour augmenter la puissance d'un site, il suffisait d'élever artificiellement la hauteur des chutes, ce qui provoquait la formation de réservoirs en amont (figure 3). Pareils dispositifs permettaient de concentrer en un endroit précis le potentiel de toute une section d'un cours d'eau, en plus d'avoir un effet bénéfique sur la régularisation du débit. Or, ces aménagements étaient susceptibles de heurter les droits des propriétaires riverains installés plus haut. La situation était d'autant plus compliquée qu'aux lots concédés par l'État depuis la fin

27. Le nombre de municipalités qui produisent elles-mêmes l'électricité pour leurs citoyens est assez élevé avant les années 1920 (en termes relatifs, il atteint un sommet en 1917, avec 29\% des localités électrifiées). Voir à ce sujet Claude Bellavance, «Réseaux, territoires et électricité», loc. cit., 399-402. 


\section{FIGURE 3}

REPRÉSENTATION SCHÉMATIQUE DE DIVERS SCÉNARIOS D'AMÉNAGEMENT D'UNE SECTION D'UN COURS D'EAU
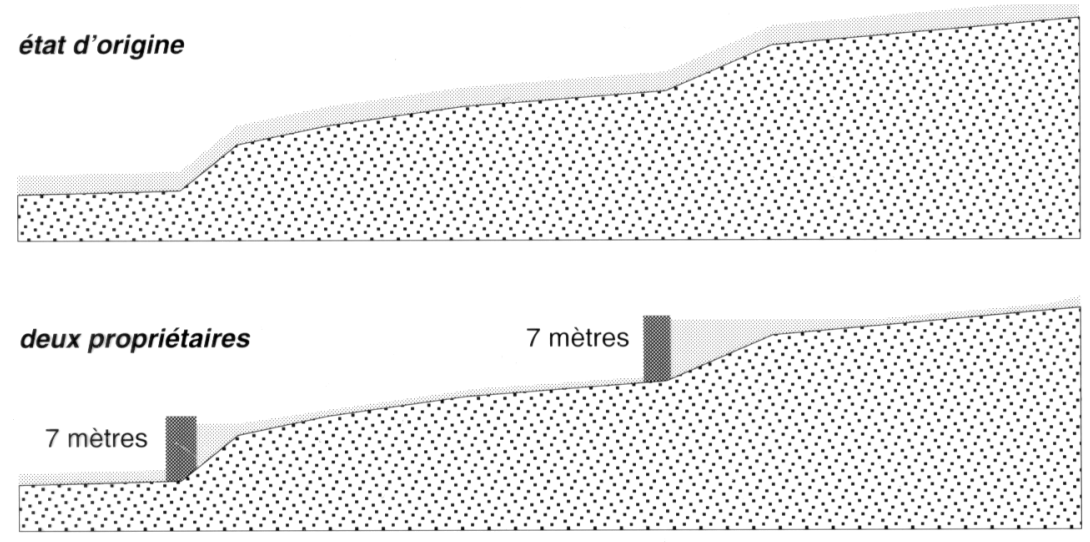

un seul propriétaire

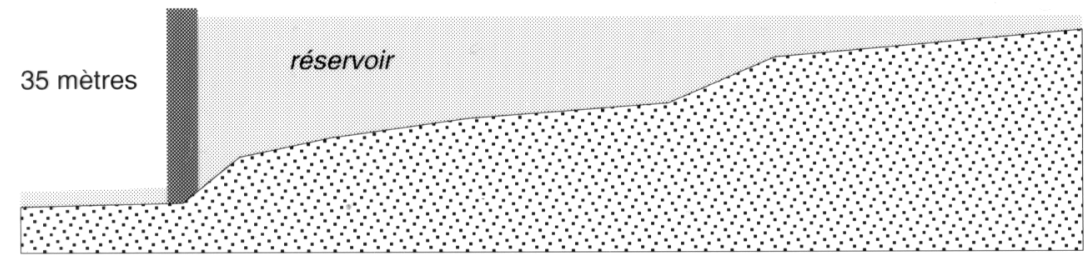

du XIXe siècle s'ajoutaient, comme on l'a vu, bon nombre de propriétés foncières achetées plus tôt. Mais quelle aubaine pour les spéculateurs tous azimuts dont les droit acquis pouvaient compromettre la réalisation de ces projets! C'est dans ce contexte qu'il faut situer l'adoption, à compter de 1909, de nouvelles mesures législatives conférant aux entreprises d'électricité des pouvoirs d'expropriation adaptés aux conditions particulières de ce service public ${ }^{28}$.

La période initiale de mise en valeur des ressources hydrauliques des rivières québécoises n'en apparaît pas moins marquée par le caractère

28. Pour faciliter la réalisation de grands ouvrages, l'État précisera la législation relative à l'expropriation, considérant dès lors nommément d'intérêt public l'aménagement de barrages de plus de $200 \mathrm{HP}$. Statuts de la Province de Québec (SDQ), 9 Édouard VII, chap. 68 (1909), «Loi concernant l'expropriation de terrains requis pour le développement et l'exploitation de certains pouvoirs hydrauliques». 
encore improvisé des décisions gouvernementales aussi bien que par l'empressement des entrepreneurs à s'approprier à bon compte des sites de grande valeur. La situation commandait des changements d'orientation de la part de l'État qui - sans rompre pour autant avec sa philosophie première - allait devoir intervenir plus directement afin de redéfinir les règles du jeu, voire pour imposer diverses restrictions. Les réformes se traduisirent notamment par le remplacement de la vente en pleine propriété par la formule du bail emphytéotique et par la mise sur pied de la Commission du Régime des eaux courantes (CEC). Avant d'analyser les conséquences d'une décennie de laxisme de l'État et d'évaluer l'impact de l'adoption du bail à long terme, il nous faut exposer le mandat ${ }^{29}$ de la nouvelle régie publique appelée à jouer un rôle de premier plan dans la rationalisation des conditions d'exploitation des rivières québécoises.

\section{B -LA COMMISSION DES EAUX COURANTES: VERS UNE GESTION UNIFIÉE DES BASSINS HYDROGRAPHIQUES}

Au tournant du siècle, un vaste mouvement d'opinion en faveur de la protection de la nature s'exprimait à l'échelle nord-américaine. Il s'agit du mouvement conservationniste, dont on connaît mieux la genèse et l'influence au Canada grâce aux travaux de Michel Girard ${ }^{30}$. Cette initiative, comme on le sait, était le résultat de la prise de conscience chez une fraction des élites nord-américaines de la dégradation des habitats naturels et de l'environnement, dans la foulée du développement rapide du capitalisme de grandes entreprises aux États-Unis. Le président T. D. Roosevelt y était associé de près. Leur position était à la fois empreinte de nostalgie - celle d'une sorte de Paradis perdu — et, par l'importance accordée à l'exploitation raisonnable des ressources naturelles, de rationalisme. Au Canada, les membres de la Commission de la conservation furent entre autres responsables de l'interdiction d'exporter la pulpe de bois. Ils ont été aussi les instigateurs d'un programme d'inventaire des ressources naturelles du domaine public. À ce titre, ils furent en quelque sorte à l'origine de la création, en 1910, de la Commission du régime des eaux courantes.

29. La CEC est certainement le moins étudié des organismes publics qui — à l'instar de la Commission des services publics, la Régie de l'électricité — ont œuvré depuis le début du siècle dans le secteur de l'électricité. On trouve à ce sujet quelques informations et remarques intéressantes dans J. H. Dales, op. cit., 30, 69 et 223.

30. Michel F. Girard, L'écologisme retrouvé. Essor et déclin de la Commission de la conservation du Canada (Ottawa, Presses de l'Université d'Ottawa, 1994), 308 p. 


\section{1- Mieux connaître la ressource pour une utilisation plus rationnelle}

Les «conservationistes» avaient déploré l'insuffisance des données disponibles sur le régime des cours d'eau canadiens (évaluation de leur dénivellation, de l'étendue de leur bassin et de leur débit etc.) ${ }^{31}$. Pallier ces lacunes, tel allait être le mandat premier imparti à la CEC dont les ingénieurs, au cours des 45 années de son histoire, allaient réaliser plusieurs centaines d'études hydrologiques. La nouvelle régie publique avait plus spécifiquement pour fonction de préciser les règles «pratiques et équitables» devant régir l'écoulement, la dérivation, l'aménagement, la distribution, l'emmagasinement et, en général, la conservation et l'administration des eaux courantes sur le territoire québécois ${ }^{32}$.

La parenté d'esprit qui régnait entre la Commission de la conservation et la nouvelle Commission du régime des eaux courantes du Québec ressort d'ailleurs avec évidence à la lecture des documents issus des deux organismes. Dans l'introduction de son premier rapport annuel, en 1912, la CEC rappelle les grandes conclusions de ses prédécesseurs et présente ses principales prises de position. Mais c'est la promotion de l'utilisation rationnelle des cours d'eau qui la préoccupe avant tout. On prend soin, dès le départ, de préciser ce qu'on entend par conservation des ressources, soit «non seulement leur protection mais aussi leur utilisation bien comprise dans l'intérêt public ${ }^{33} »$. Cette orientation transparaît d'ailleurs dans le choix de ses membres: les commissaires Ernest Bélanger et William Bishop, tous deux ingénieurs civils, et son président, Simon-Napoléon Parent, ancien ministre des Terres et Forêts, puis Premier ministre du Québec de 1900 à 1905, chaud partisan de l'hydroélectricité, mais également l'un des responsables de la politique gouvernementale de «laissezfaire» précédemment évoquée.

Quelles étaient donc les priorités de la CEC au moment de sa formation? La première mission des commissaires fut de définir le statut juridique des rivières québécoises et de proposer un cadre pour leur classification. La tâche était ardue en raison des lacunes de la législation et des interprétations contradictoires auxquelles elle avait donné lieu. Après avoir pris connaissance des expériences américaine et française,

31. L. G. Denis, et A. V. White (Commission de la Conservation du Canada), Les forces hydrauliques du Canada (Ottawa, Mortimer, 1911), $397 \mathrm{p}$.

32. Elle devait en outre examiner la législation relative aux cours d'eau et procéder au classement des rivières selon qu'elles soient navigables et flottables ou non navigables et non flottables. CEC, Rapport annuel, 1912, 4-7, et $S D Q, 1$ Georges V, chap. 5 (1910), «Loi autorisant l'organisation d'une commission chargée de proposer des règles pour fixer le régime des eaux courantes».

33. CEC, Rapport annuel, 1912, 13. 
puis cerné plus largement les grandes tendances internationales ${ }^{34}$, la Commission concluait qu'il fallait modifier en profondeur les principes régissant l'usage des cours d'eau. Pour rompre avec la situation actuelle, alléguait-elle, il faut «un principe sûr et logique» qui favorise une gestion «rapide, uniforme, certaine et formelle ${ }^{35}$ ». Dans cette perspective, elle recommandait au gouvernement de décréter comme relevant d'office du domaine public le lit des 181 rivières dont le bassin hydrographique excédait 300 milles carrés. En outre, une disposition particulière étendait la portée de cette recommandation aux cours d'eau dont la dimension des bassins était encore inconnue ${ }^{36}$.

\section{2- L'État entrepreneur: la formation du réservoir Gouin}

Au tout début de leur mandat, les commissaires se tournèrent vers ce qui allait devenir le grand projet de la CEC: l'érection d'un barrage pour la régularisation du Saint-Maurice. Dès 1912, la création du réservoir Gouin était mise de l'avant comme première priorité. Pour justifier leur hâte, ceux-ci prétendirent que l'expérience acquise pourrait être appliquée ailleurs. Cet argument ne convainc toutefois qu'à moitié. Déjà, les grandes entreprises détentrices de sites en région avaient amorcé la construction de barrages sur un affluent de la rivière et elles comptaient obtenir l'assentiment de l'État pour créer un vaste réservoir en HauteMauricie. Les témoignages retranscrits dans les rapports de la $\mathrm{CEC}^{37}$ donnent à penser que le projet mis de l'avant par Shawinigan Water and Power, Laurentide Paper de Grand-Mère et Brown Corporation de La Tuque suscitait l'opposition du milieu. Aussi, les dirigeants de ces grandes entreprises se montraient-ils tout à fait favorables à sa prise en charge par un organisme public, auquel elles verseraient des droits supplémentaires pour augmenter la puissance de leur centrale.

Mais pourquoi donc réaliser un tel ouvrage? La réponse réside en partie dans les récents progrès de l'hydrologie ${ }^{38}$ qui, vers 1910 , mirent à l'ordre du jour des projets de formation de réservoirs un peu partout dans les pays industrialisés. C'est qu'on savait maintenant qu'en constituant un réservoir, on pouvait hausser sensiblement la puissance tirée d'un cours

34. CEC, Rapport annuel, 1913, 32-37.

35. Ibid., 46.

36. L'État n'adoptera pas le classement proposé en 1913 par les ingénieurs de la CEC. La dernière mention de ce projet figure au rapport de 1921. CEC, Rapport annuel, 1921, 7.

37. CEC, Rapport annuel, 1912, 95-110.

38. Voir à ce sujet Norman Alfred Fisher Smith, Man and Water: a History of Hydro-Technology (New York, Scribner, 1975), 239 p.; ainsi que, du même auteur A History of Dams (London, P. Davies, 1971), 279 p. 
d'eau. Considérons le problème de plus près. Au printemps, avec la fonte des neiges, les rivières se gonflent: la période de crue libère des quantités formidables d'énergie. L'hiver et quelques semaines durant l'été, au contraire, le débit des rivières est souvent fortement diminué en l'absence de précipitations. Or, l'écart entre les deux situations peut être considérable. Le régime naturel de la rivière Saint-Maurice, par exemple, a fluctué entre 6000 et 200000 pieds cube en 1913 (voir les courbes de précipitations). De telles variations posaient de sérieux problèmes sur le plan économique. Car si l'on installe des équipements capables d'exploiter le débit des chutes lors de la crue, ces derniers seront sous-utilisés le reste de l'année. On peut au contraire décider de construire une centrale en fonction du débit minimal, mais au prix d'un gaspillage d'énergie la majeure partie du temps. À cette époque, les centrales sont généralement conçues pour obtenir un rendement de l'ordre de 60 à $80 \%{ }^{39}$; elles sont surdimensionnées par rapport aux périodes de basses eaux, afin de tirer profit de l'énergie libérée au cours des autres mois. C'est dire que plus un régime d'un cours d'eau est contrasté, moins il sera rentable d'exploiter l'ensemble de son potentiel énergétique. À l'inverse, un cours d'eau comme le Saint-Laurent, dont le rapport entre les débits maximal et minimal n'est que de l'ordre 1 à 2 apparaîtra particulièrement avantageux ${ }^{40}$. La découverte de l'heure - il serait plus juste de parler d'application, sur une large échelle, de principes déjà connus — c'est qu'en retenant l'eau à l'aide de barrages situés à la tête des rivières, on pouvait dès lors envisager la construction de très grandes centrales hydroélectriques et bénéficier d'économie d'échelle substantielles.

Le réservoir Gouin allait permettre de régulariser le cours du SaintMaurice à environ 17000 pieds cubes/seconde, c'est-à-dire de tripler son débit minimal. Après les études hydrologiques menées par les experts au service de la Commission, l'élaboration des plans et devis et les appels d'offre, les travaux débutèrent en 1915. C'est l'ancêtre de Shawinigan Engineering (elle-même filiale de Shawinigan Water and Power), la compagnie St.Maurice Construction qui, au nom de la CEC, en prit la responsabilité. Ses ingénieurs avaient à leur actif l'aménagement de Les Cèdres à proximité de Montréal, la plus grande centrale au monde à cette époque. Le barrage complété en décembre 1917 allait être considéré comme une

39. Il s'agit du rapport entre l'énergie réellement produite et celle hypothétiquement obtenue sur la base d'un fonctionnement à pleine capacité tout au long de l'année.

40. La faible dénivellation de ses rapides soulève cependant d'autres difficultés. Pour capter l'énergie d'un cours d'eau comme le Saint-Laurent, il est nécessaire, en effet, d'utiliser des turbines adaptées. On désigne ce type d'aménagement sous le vocable «centrale au fil de l'eau». 


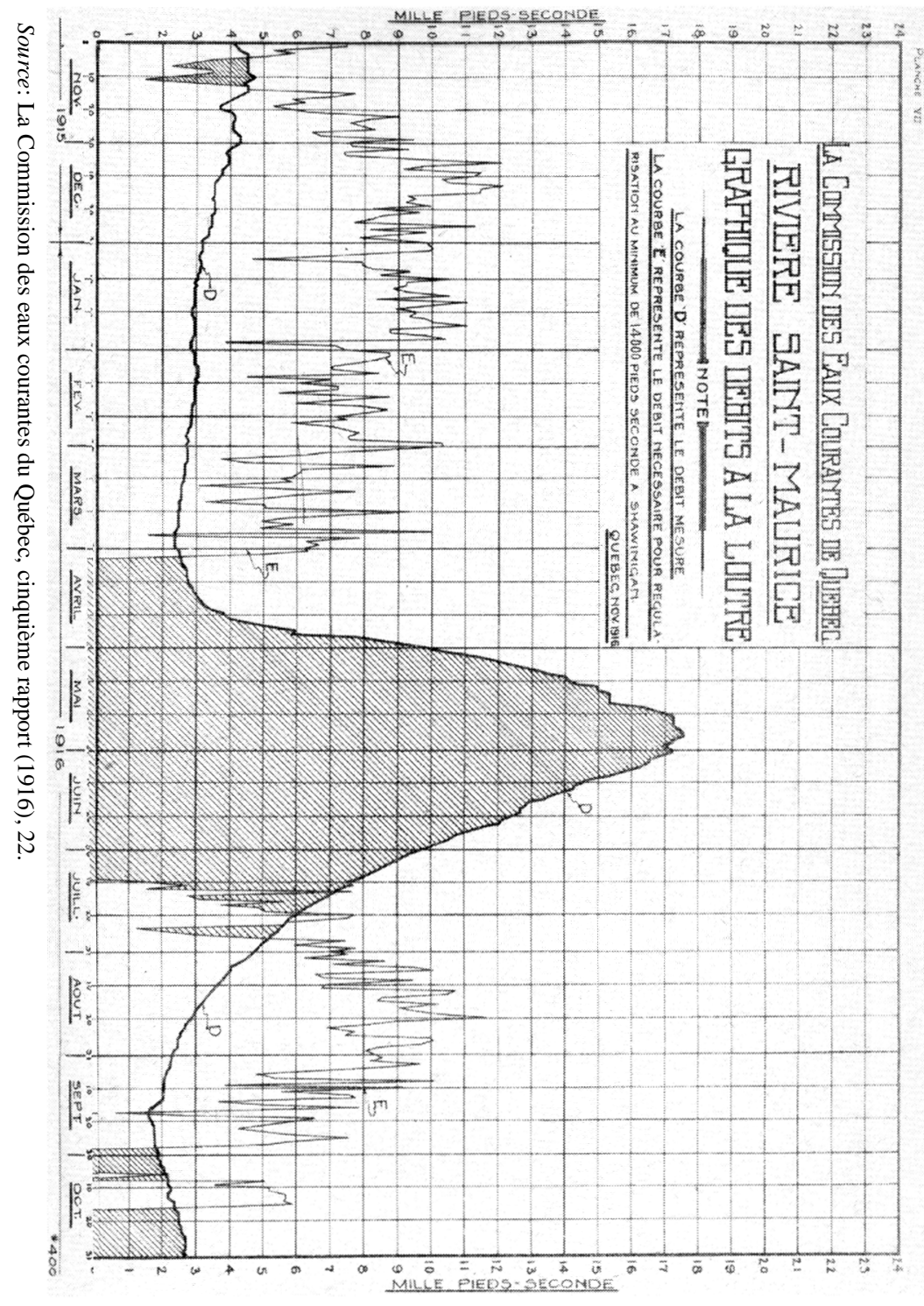

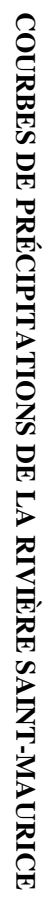


réalisation majeure à l'échelle internationale ${ }^{41}$. Il mettait clairement en évidence les avantages de la participation de l'État à la conception et à la réalisation d'ouvrages de retenue de grande ampleur, de même qu'à sa prise en charge subséquente, au nom de l'intérêt général.

\section{3- La régularisation de la rivière Saguenay: un rôle quasi symbolique pour l'État}

D'autres projets de régularisation furent soumis à la CEC pendant ses premières années d'existence, notamment à la source des rivières SaintFrançois, Métis et Sainte-Anne-de-Beaupré, de même qu'au lac Kénogami. La plupart étaient plutôt modestes, quoique leur impact sur les milieux naturels - modification des écosystèmes, réduction des risques d'inondation etc. - pût être significatif.

$\mathrm{Au}$ lendemain de l'inauguration des travaux en Haute-Mauricie, c'est du côté du Saguenay_Lac-Saint-Jean que l'attention des commissaires de la CEC allait se tourner pour un temps. À l'instigation du département des Terres et Forêts, la Commission fut chargée, en 1915, d'établir la capacité du lac Saint-Jean comme réservoir d'emmagasinement. Comme pour la Saint-Maurice, la CEC était appellée à prendre le relais dans un dossier déjà en marche. Quebec Development — propriété de J. B. Duke, l'une des figures dominantes du capitalisme américain à cette époque ${ }^{42}$ — qui détenait les droits d'exploitation des deux principaux sites du Saguenay, avait procédé depuis

41. Le réservoir Gouin, avec une capacité de 160 millions de pieds cubes et une superficie de 300 milles carrés, était alors le plus grand au monde. Il coûta environ 2,5 millions de dollars, une somme relativement importante pour l'époque (à titre indicatif, le budget annuel de la Province de Québec était de l'ordre de 11,6 millions de dollars lorsque débutèrent les études sur le terrain, en 1912, et de 14,4 millions de dollars au moment de son inauguration, en 1917). CEC, Rapport annuel, 1918, 32-34 et Annuaire statistique du Québec, 1918.

42. Dans une thèse récente, David Massell a reconstitué avec minutie l'histoire de l'aménagement des sites hydroélectriques du Saguenay. Recourant à une documentation inédite, il a notamment levé le voile sur les enjeux des négociations entre le grand capital américain et le service hydraulique du Québec. Il appert que si le magnat du tabac J. B. Duke détenait au début des années 1910 des droits en pleine propriété sur les sites Isle Maligne et Chute à Caron, il devait cependant obtenir de l'État québécois le droit d'augmenter le niveau du lac. Une première phase de négociations se termina en 1916 par l'abandon d'un projet d'implantation, au Saguenay, d'une usine d'acide nitrique (DuPont). L'ingénieur A. Amos, du Service hydraulique du Québec, qui avait eu la responsabilité du dossier et l'appui de ses supérieurs, avait alors exigé de Duke et de ses associés qu'ils acceptent de payer des redevances pour le surplus de puissance que la régularisation de la rivière leur procurerait. Après la guerre, un nouveau contexte politique plus favorable à l'entreprise américaine prévalut. Duke parvint cette fois à s'entendre avec les représentants du pouvoir politique, non sans s'être assuré que les discussions fussent menées au plus haut niveau (directement avec le nouveau Premier ministre L.-A. Taschereau). La permission de hausser le niveau des eaux du lac Saint-Jean fut finalement accordée en 1922; d'autres contraintes furent levées par l'État lorsque Alcoa s'apprêta à reprendre les actifs saguenayens de Duke. David P. Massell, op. cit. Voir aussi José E. Igartua, Arvida au Saguenay: naissance d'une ville industrielle (Montréal, McGill-Queen's University Press, 1996), 13-31 et id., «"Corporate" Strategy and Locational Decision-Making: the Duke-Price Alcoa Merger, 1925», Revue d'études canadiennes/Journal of Canadian Studies, 20,3 (automne 1985): 82-101; Claude Bellavance, Shawinigan Water and Power, 1898-1963, op. cit., 97-102. 
quelques années à divers relevés hydrologiques. Les ingénieurs de la CEC durent examiner ici quatre scénarios. Un premier prévoyait de régulariser le cours du Saguenay à 22000 pieds cubes par seconde. Pour y parvenir, un barrage aurait été construit qui eût haussé le niveau du lac Saint-Jean de quelque neuf pieds. Un deuxième plan prévoyait une élévation de 15 pieds pour un débit de 25000 pieds cubes. En montant le niveau du lac de 20 pieds et demi, on maintiendrait, croyait-on, un débit de 28000 pieds cubes. Enfin, en augmentant le niveau des eaux de 30 pieds, il était théoriquement possible d'obtenir 33000 pieds cubes. Le rapport de l'ingénieur de la Régie mettait cependant en garde les commissaires à propos de l'impact environnemental de ces hypothèses. Avec le premier scénario envisagé, une portion des terres arables recouvertes au printemps demeurerait après coup immergée; beaucoup plus ambitieux, le dernier projet, lui, menacerait jusqu'aux quais du gouvernement fédéral!

Bien que la question du lac Saint-Jean et de ses tributaires fut à nouveau examinée par la CEC en 1921, les démarches précédemment entreprises ne se soldèrent pas, dans ce cas, par sa prise en charge de l'aménagement des ouvrages de régularisation. Quebec Development, puis Duke-Price Power et Alcan furent plutôt confirmés en tant que maîtres d'œuvre des grands chantiers de la région ${ }^{43}$. C'est à eux que devait incomber la responsabilité d'en contrôler le débit. Le recul de l'État, soulignons-le, a eu des conséquences considérables. Le dossier des forces hydrauliques du Saguenay_Lac-Saint-Jean devait d'ailleurs rapidement s'envenimer pour le gouvernement Taschereau. En 1924, des précipitations exceptionnelles avaient provoqué de fortes inondations, entraînant de lourds dommages aux propriétaires riverains. Leur situation allait encore se dégrader lorsque, deux ans plus tard, Duke-Price obtint enfin la permission de rehausser le niveau du lac: face au nouveau danger, un groupe de citoyens menaça même de dynamiter les ouvrages de régulation. Afin d'apaiser la population, Duke-Price dut consentir à ce que la CEC veille ellemême à la gestion du barrage de la Petite décharge ${ }^{44}$. Il ne s'agissait cependant que d'une responsabilité toute symbolique qui ne remettait nullement en cause la mainmise de l'entreprise privée sur le bassin hydrographique saguenayen.

En fait, l'épisode du Saguenay_Lac-Saint-Jean marque un recul de l'État comme intervenant dans l'aménagement de bassins hydrographiques. À partir du milieu des années 1920, la CEC allait en pratique céder la réalisation

43. David P. Massell, op. cit., 361-412.

44. «La crue extraordinaire des eaux au printemps de 1928 a donné lieu à beaucoup de discussion, plus particulièrement dans la région du Lac St-Jean. En vue de faire disparaître dans le public l'idée que la compagnie propriétaire du barrage à l'Ile Maligne était libre d'opérer ce barrage à sa guise, le gouvernement a demandé à notre Commission de prendre contrôle durant les périodes d'inondation au printemps. Ce contrôle a commencé dès février 1929. [...]», CEC, Rapport annuel, 1929, 13. 
des nouveaux ouvrages de régulation au secteur privé. La création de réservoirs aux sources de la Gatineau et de La Lièvre en Outaouais fut, en effet, laissée aux entreprises qui allaient aménager des centrales sur ces rivières. Certes, lesdites sociétés construisirent les ouvrages de régularisation conformément aux devis de l'organisme public qui en conservait la propriété et la gestion formelle. Toutefois, il ne semble pas que ce soient les ingénieurs de la CEC qui en élaborèrent les plans. La Commission aurait plutôt choisi de confier la tâche à des hydrauliciens «indépendants». Plus important encore, contrairement au cas de la Haute-Mauricie, ce furent les entreprises privées qui assumèrent ici l'ensemble des coûts de construction. On peut dire qu'à partir de cette époque, les conditions de création des réservoirs à la tête des grands cours d'eau québécois se rapprochaient singulièrement de celles prévalant pour la location des sites de centrales: l'État demeurait propriétaire, mais il n'assumait qu'un rôle nominal dans la mise en valeur intégrée des potentiels ${ }^{45}$. Comme nous le verrons maintenant, la politique gouvernementale en matière de mise en valeur des ressources hydrauliques s'orientait alors résolument vers le soutien à la formation de monopoles régionaux de la production d'électricité, moyennant un partage des revenus tirés de leur exploitation.

\section{C- UN NOUVEAU RÉGIME D'ALIÉNATION DE LA RESSOURCE}

\section{1- Des revenus désormais substantiels pour l'État, mais des potentiels encore largement sous-évalués}

La politique libérale que pratiquait l'État québécois depuis la fin du XIX ${ }^{\mathrm{e}}$ siècle conduisit, comme nous l'avons vu précédemment, à un développement peu harmonieux de la ressource. En une dizaine d'années seulement, quantité de chutes et de rapides passèrent entre les mains de propriétaires dont les intérêts s'avéraient difficilement conciliables, quand ils n'étaient pas carrément contradictoires. Certes, on avait parfois exigé de l'acquéreur qu'il procède à l'aménagement du site dans des délais fixes et qu'il y engage des fonds relativement élevés ${ }^{46}$. On hésitait cependant à recourir aux tribunaux pour faire

45. CEC, Rapports annuels, 1925 à 1933.

46. À propos de la vente en propriété de rapides situés sur la rivière Métabetchouan on peut lire: «L'acquéreur devra dépenser d'ici à 2 ans la somme de $\$ 150,000.00$ pour développer les forces hydrauliques. Si cette condition n'est pas remplie, l'acquéreur devra payer en plus la somme de $\$ 5,000.00$.» On trouve les mêmes exigences pour la chute à Caron au Saguenay: «Dépenser $\$ 1,000,000.00$ pour développer et exploiter les forces hydrauliques. À défaut de ce faire, une somme de $\$ 6,000.00$ sera exigée en plus du prix de vente.» Une date pour l'exécution des travaux est précisée dans 14 des 92 ventes par lettres patentes de la période 1896-1913. Le document mentionne explicitement que les engagements du cessionnaire n'ont pas été respectés dans douze cas. Ministère des Terres et Forêts, Service hydraulique (1914), Tableau des Forces Hydrauliques concédées par la Province de Québec, du $1{ }^{\mathrm{er}}$ juillet au 31 décembre 1913. 
annuler la vente et l'octroi de lettres patentes venait souvent consacrer l'aliénation définitive d'un site sans que celui-ci n'ait encore été mis en valeur ${ }^{47}$. Le régime de la vente en pleine propriété prévalut jusqu'à la fin des années 1900 pour céder la place au bail à long terme. Des baux furent contractés dès $1906^{48}$, alors que A. E. de Lorimier de Montréal s'engageait à aménager la première chute de la rivière Manicouagan ${ }^{49}$. Mais c'est à partir de 1910 que le bail emphytéotique devint véritablement la norme (figure 4). Cette annéelà, on vit notamment l'octroi des droits d'exploitation des rapides Les Cèdres à l'ouest de Montréal; il s'agissait du plus grand projet hydroélectrique au monde jamais conçu.

Si la location des sites hydrauliques rendait beaucoup plus aisée la révocation des droits du concessionnaire en cas de dérogations aux clauses du contrat, elle n'en était pas moins bien vue parmi les industriels et les financiers influents engagés dans les grands projets hydroélectriques de l'heure puisqu'elle disqualifiait, à toutes fins utiles, les petits entrepreneurs et les spéculateurs. L'État allait exiger en retour de profiter lui aussi de l'exploitation de la ressource sous la forme d'une véritable rente énergétique.

Grâce aux données colligées par la CEC et par le Service hydraulique canadien, l'État québécois était maintenant en mesure, en effet, d'exiger des sommes beaucoup plus élevées pour l'exploitation des sites aliénés et la régulation des cours d'eau. Ainsi, entre 1914 et 1927, les redevances passèrent de $15000 \$$ à plus d'un million de dollars, ce dernier montant représentant alors 3,1\% des recettes ordinaires de la Province (figure 5). Contrairement à l'ensemble des encaisses qui diminuèrent de plus du tiers en cette période de crise économique, les revenus tirés de l'exploitation

47. Vers 1900, plusieurs propriétaires de sites hydrauliques firent des démarches auprès du ministère des Terres et Forêts afin de faire sanctionner leurs droits et, à certaines occasions, pour obtenir des titres complémentaires. C'est le cas, notamment, des rapides des Grès, près de TroisRivières, et des chutes de Grand-Mère sur la Saint-Maurice, de même que de forces hydrauliques exploitées depuis les années 1870 par John McLaren Co. Ltd sur la Lièvre près de Buckingham. Ibid. et Documents de la Session du Québec (DSQ), 1901, «Correspondance concernant les pouvoirs d'eau des Grès sur le St-Maurice» et Documents de la Session du Québec, 1900, «Copie de tous les arrêtés en Conseil et de toutes les décisions des Terres et Forêts autorisant la vente des pouvoirs d'eau depuis le $1^{\text {er }}$ juillet 1897, Chutes de Grand'Mère», Ibid., «Rapides des Chats, rivière Outaouais».

48. En 1899, avant de s'en porter acquéresse, Upper Ottawa Improvement Co. tente d'obtenir la location de Rapides des Chats. La correspondance révèle le peu d'intérêt du ministère des Terres et Forêts pour cette formule à cette époque. $D S Q$ 1900, «Copie de tous les arrêtés en Conseil et de toutes les décisions des Terres et Forêts autorisant la vente des pouvoirs d'eau depuis le $1^{\text {er }}$ juillet 1897, Rapides des Chats, rivière Outaouais».

49. En 1919, la concession passe à Manicouagan \& English Bay Export Co. Ltd. Département des Ressources hydrauliques, Tableau des forces hydrauliques de la Province de Québec concédées 1) par lettres patentes; 2) sous baux emphytéotiques, à la date du $1^{\mathrm{er}}$ janvier 1947. 
FIGURE 4

LES SITES CONCÉDÉS ENTRE 1896 ET 1914, NOMBRE ET PUISSANCE ESTIMÉE SELON LE MODE D'ALIÉNATION (D'APRÈS LE RELEVÉ OFFICIEL DE 1914)

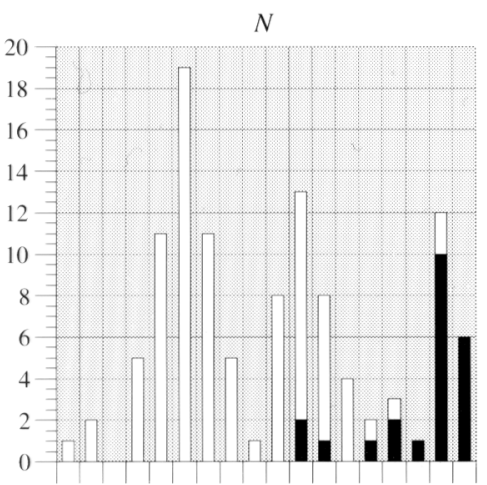

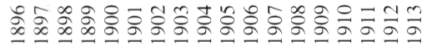

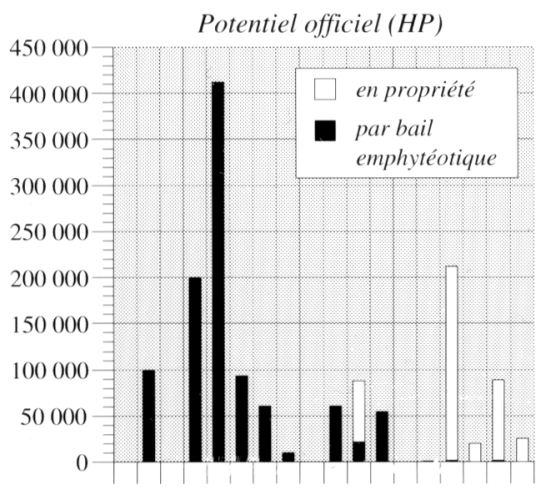

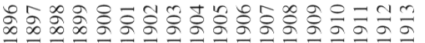

Source: Ministère des Terres et Forêts, Service hydraulique (1914), Tableau des forces hydrauliques concédées par la Province de Québec, du $1^{\text {er }}$ juillet au 31 décembre 1913.

des cours d'eau continuèrent d'augmenter. Nous avons calculé qu'en 1933, presque un dollar sur vingt provenait de la ressource hydraulique ${ }^{50}$.

En privilégiant cette formule, l'État visait non seulement à constituer une rente énergétique, mais aussi à corriger les problèmes hérités d'une dizaine d'années de laissez-faire. Dans quelle mesure la réforme lui conférait-elle un outil d'intervention efficace pour orienter le développement de l'industrie de l'électricité? Combien de ces sites allaient être aménagés dans des délais acceptables? Et, plus prosaïquement, allait-on désormais révoquer les titres des concessionnaires qui n'avaient pas honoré leurs engagements? Pour répondre à ces questions, il nous faut prendre en considération une plus longue période d'observation. Nous recourerons maintenant à un relevé semblable à celui qui nous avait jusqu'à présent servi de référence, mais datant celui-là de 1947, de même qu'à un inven-

50. Les revenus du ministère des Terres et Forêts pour l'exercice budgétaire 1932-1933 s'établissaient à un peu plus de 2,7 millions de dollars, ce qui en faisait la sixième source en importance, après les taxes sur l'essence (5,3 millions \$), les droits sur les véhicules (5 millions \$), la vente d'alcool (4,7 millions \$), les taxes sur les corporations (3,3 millions \$), les droits sur les successions (3 millions \$). Un peu plus du tiers de cette somme provenait alors de l'exploitation des ressources hydrauliques, le reste relevant de l'exploitation de la forêt. En 1933-1934, la part de l'hydraulique atteindra $44 \%$ des recettes du ministère en raison d'une baisse importante de l'activité de l'industrie forestière. ASQ et Comptes publics de la Province de Québec, années concernées. 
FIGURE 5

\section{LES REDEVANCES PERCCUES POUR L'EXPLOITATION DES RESSOURCES HYDRAULIQUES, EN DOLLARS COURANTS ET EN POURCENTAGE DES RECETTES ORDINAIRES DE LA PROVINCE DE QUÉBEC, 1914-1941}

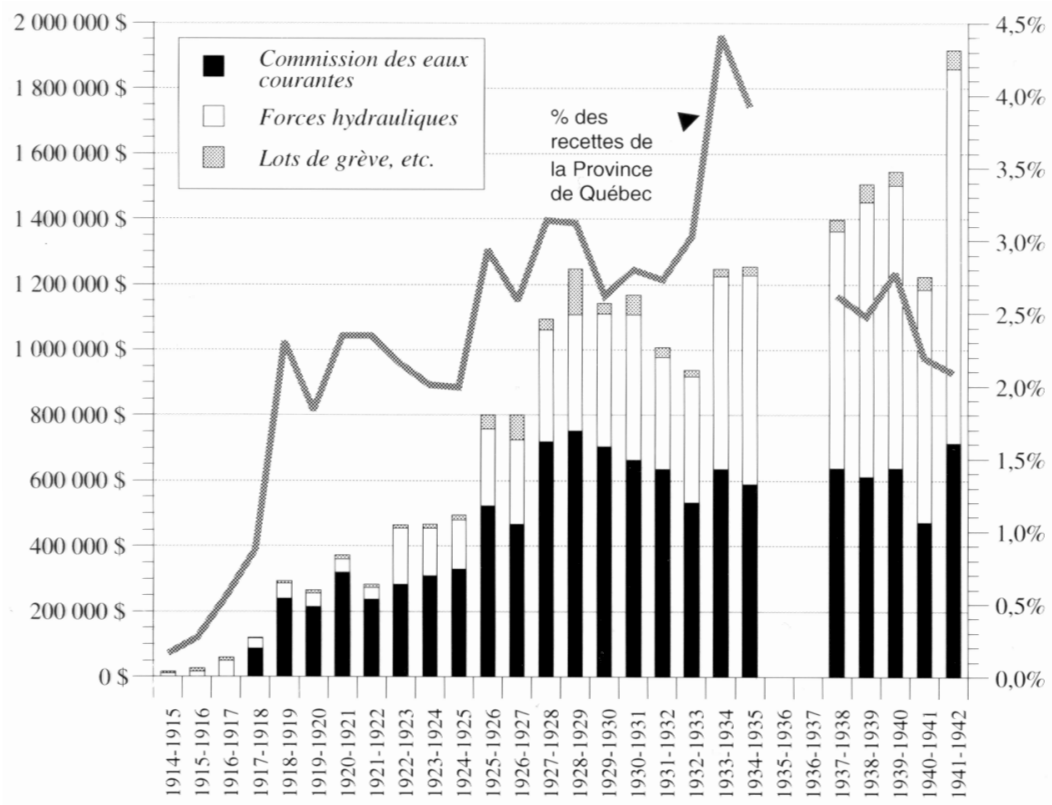

Sources: Rapports annuels de la Commission des eaux courantes, Rapports annuels du Service hydraulique du ministère des Terres et Forêts et Annuaires statistiques du Québec, années concernées.

taire exhaustif des forces hydrauliques (concédées ou non) préparé en 1928 par le Service hydraulique fédéral avec la collaboration de la CEC et du département des Terres et Forêts provincial ${ }^{51}$.

Observons d'abord les données actualisées du relevé de 1947 (figure 6). Selon cette source, environ 2,2 millions de HP sont concédés entre 1896 et 1913 (1,3 million de HP pour les seules années 1900 et 1901)

51. À la demande de l'Assemblée législative, des listes des pouvoirs d'eau concédés ont été constituées en 1907, 1914, 1923 et 1947. En outre, des relevés partiels ont été publiés à diverses reprises dans les Documents de la Session du Québec. Le relevé de 1947 a été retenu parce qu'il livre un bilan détaillé des cessions de sites au moment où s'amorce une toute nouvelle période de l'histoire de l'électricité au Québec, période marquée par l'arrivée d'Hydro-Québec et par la réalisation des projets d'aménagement des bassins de la Côte-Nord (rivières Bersimis, Manicouagan-Outarde etc.). Bien que ces tableaux soient de facture similaire, les données présentées ne sont pas directement comparables. Comme on le verra, les différences les plus marquantes tiennent à l'appréciation des potentiels. 
FIGURE 6

LES SITES CONCÉDÉS ENTRE 1896 ET 1947, NOMBRE ET PUISSANCE ESTIMÉE SELON LE MODE D'ALIÉNATION (D'APRÈS LE RELEVÉ OFFICIEL DE 1947)

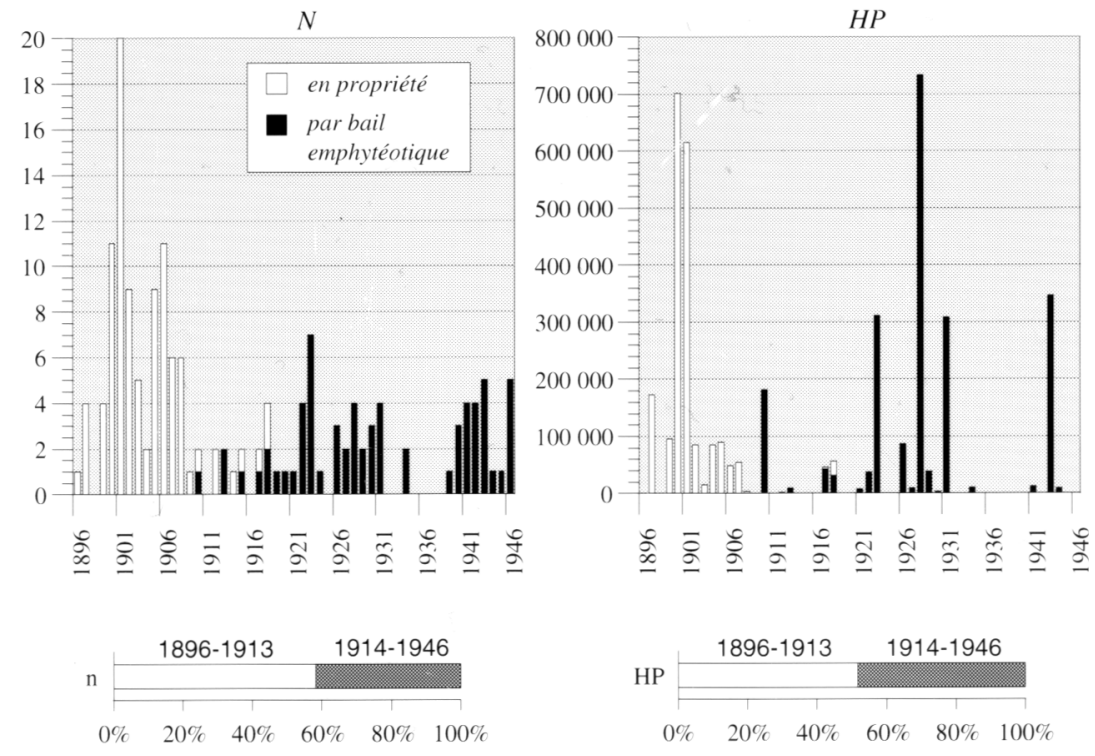

Sources: Département des Ressources hydrauliques, Tableau des forces hydrauliques de la Province de Québec concédées 1) par lettres patentes; 2) sous baux emphytéotiques à la date du 1er janvier 1947.

contre seulement 2 millions pour les 32 années suivantes. Au total, 163 chutes et rapides auraient été vendus ou loués depuis 1896, cumulant une puissance estimée à un peu plus de 4 millions de HP. Au terme de cette première période, six sites sur dix avaient donc été définitivement aliénés. Cela représente plus de la moitié du potentiel hydraulique en cause!

L'analyse révèle par ailleurs que les réserves énergétiques des cours d'eau québécois ont été hautement sous-évaluées pendant la phase initiale de l'histoire de l'électricité. On pourra constater l'ampleur du phénomène en examinant le tableau 1, qui dresse la liste des sites de $10000 \mathrm{HP}$ et plus concédés avant $1914^{52}$. La comparaison entre les évaluations provin-

52. Ces sites correspondent à la catégorie $\mathrm{C}$ dans la précédente analyse de répartition. Rappelons que cette catégorie compte pour plus de $90 \%$ de la puissance concédée avant 1914. Le jumelage systématique de ces trois sources est actuellement en cours, un travail complexe qui nécessitera des recoupements avec d'autres types de documents et qui devra être étendu à la période 1945-1965. 
ciales de 1914 et celles effectuées par le Service hydraulique fédéral en 1928 montre les écarts les plus considérables (117\% si l'on considère l'ensemble de ces chutes et rapides ${ }^{53}$ ).

\section{2- Les monopoles régionaux et la rationalisation de l'exploitation des bassins hydrographiques}

Le mode d'aliénation de la ressource en vigueur avant 1914 a favorisé l'appropriation de sites hydrauliques par des entrepreneurs qui n'étaient pas dotés des capitaux nécessaires à leur mise en valeur ou par des spéculateurs à l'affût des bonnes occasions. À cette époque, comme nous l'avons signalé, l'État se montrait plutôt conciliant envers les concessionnaires qui laissaient leurs propriétés inexploitées $(80 \%$ des sites en 1914) et ce, même longtemps après leur acquisition. La situation, cependant, s'est nettement améliorée une trentaine d'années plus tard, alors que $72 \%$ des potentiels sont aménagés. Le revirement est encore plus frappant en ce qui concerne les sites de $10000 \mathrm{HP}$ et plus: des 54 chutes ou rapides en cause, seulement sept sont restés en plan en $1947^{54}$. Pourtant, il ne faudrait pas voir dans cet effort de rationalisation des conditions d'exploitation de la ressource le seul signe d'une application plus stricte par l'État des termes des contrats. Les documents officiels révèlent bien quelques cas de révocation des droits de propriétaires ou de locataires, mais leur nombre est trop limité pour expliquer un redressement de pareille ampleur.

L'examen du relevé de 1947 montre qu'en fait, c'est le rachat des titres par des entreprises d'électricité qui s'est révélé le moyen le plus efficace pour assurer la mise en valeur des potentiels énergétiques déjà concédés. Ainsi, au cours de la décennie 1920, Shawinigan Water and Power prit le contrôle du bassin de la Saint-Maurice en acquérant les droits d'exploitation des chutes de La Gabelle, des Forges et de GrandMère; Alcoa et sa filiale, Alcan, s'approprièrent le potentiel du Saguenay; Gatineau Power ceux des rivières Outaouais et Gatineau; et

53. La moyenne des écarts s'établit à $196 \%$ (soit un rapport de un à trois). Le tableau de 1928, à l'encontre des documents préparés pour la législature québécoise, n'expose pas une situation de fait (les potentiels stipulés dans les contrats ou les baux), mais repose plutôt sur les données tirées des plus récentes études hydrologiques de l'époque. Signalons que le cas des rapides de Lachine, près de Montréal (évalués à 700000 HP au relevé de 1928), n'a pas été considéré dans nos calculs puisqu'on semble se référer ici à toute une section du fleuve plutôt qu'à un rapide localisé et bien circonscrit, comme on le fait dans les documents d'origine provinciale.

54. Six d'entre eux ont d'ailleurs été aliénés au tout début du siècle. Le septième est un bail liant le gouvernement du Québec et Hydro-Ontario à la suite d'une entente (1943) entre les deux provinces voisines pour le partage des ressources de la rivière Outaouais. 
TABLEAU 1

LES SITES OFFRANT UN POTENTIEL SUPÉRIEUR À 10000 HP CONCÉDÉS AVANT 1914 (SELON LES ÉVALUATIONS OFFICIELLES DE 1914, 1928 ET 1947)

\begin{tabular}{|c|c|c|c|c|c|}
\hline & \multicolumn{2}{|c|}{$\begin{array}{l}\text { Documents } \\
\text { du Québec }\end{array}$} & \multirow{2}{*}{$\begin{array}{c}\text { Service } \\
\text { hydraulique } \\
\text { fédéral } \\
1928\end{array}$} & \multicolumn{2}{|c|}{$\begin{array}{c}\text { Écarts par rapport } \\
\text { à l'évaluation de } \\
1914\end{array}$} \\
\hline & 1914 & 1947 & & $\begin{array}{c}\text { Q.1947 } \\
\%\end{array}$ & $\begin{array}{c}\text { F. } 1928 \\
\%\end{array}$ \\
\hline Saguenay/Chute à Caron 2 & 200000 & 270000 & 505400 & 35 & 153 \\
\hline Saguenay/Île Maligne & 200000 & 200000 & 278000 & 0 & 39 \\
\hline Saint-Laurent/Les Cèdres & 180000 & 180000 & 197400 & 0 & 10 \\
\hline Saguenay/Chute à Caron 1 & 100000 & 100000 & 505400 & 0 & 405 \\
\hline Saint-Maurice/Shawinigan & 100000 & 160000 & 228700 & 60 & 129 \\
\hline Saint-Maurice/Grand'Mère & 66000 & 66000 & 117400 & 0 & 78 \\
\hline Manicouagan/Premières Chutes & 65000 & 65000 & 93051 & 0 & 43 \\
\hline Outaouais/Rocher Fendu & 52000 & 50000 & 131090 & 4 & 152 \\
\hline Saint-Maurice/La Tuque & 50000 & 50000 & 115000 & 0 & 130 \\
\hline Lièvre/Grandes Chutes & 34600 & 41000 & 41291 & 18 & 19 \\
\hline Rivière des Quinze 1 & 30000 & 30000 & & 0 & \\
\hline Rivière des Quinze 2 & 30000 & 30000 & & 0 & \\
\hline Gatineau/Chute Chelsea & 27000 & 100000 & 86400 & 270 & 220 \\
\hline Gatineau/Chute Paugan & 27000 & 35000 & 123000 & 30 & 356 \\
\hline Outaouais/Carillon & 26600 & 26600 & 144700 & 0 & 444 \\
\hline Outaouais/Quinze & 26200 & 42000 & 62860 & 60 & 140 \\
\hline Saint-Laurent/Lachine & 21600 & 21600 & & 0 & \\
\hline Saint-Laurent/Île au Héron & 20000 & 20000 & & 0 & \\
\hline Chicoutimi (inférieure) & 17630 & 31770 & 51187 & 80 & 190 \\
\hline Outardes/Premières Chutes & 14000 & 14000 & 70159 & 0 & 401 \\
\hline Pentecôte & 10000 & 14000 & 22101 & 40 & 121 \\
\hline Gatineau/Farmers Rapid & 10000 & 10000 & 59000 & 0 & 490 \\
\hline Total & 1307630 & 1556970 & 2832139 & 19 & 117 \\
\hline
\end{tabular}

Sources: Ministère des Terres et Forêts, Service hydraulique (1914), Tableau des forces hydrauliques concédées par la Province de Québec, du $1^{\text {er }}$ juillet au 31 décembre 1913. Département des Ressources hydrauliques, Tableau des forces hydrauliques de la Province de Québec concédées 1) par lettres patentes; 2) sous baux emphytéotiques à la date du $1^{\mathrm{er}}$ janvier 1947. Ministère de l'Intérieur, Ottawa, Service des forces hydrauliques, Département des Terres et Forêts, Québec, Commission des eaux courantes et Service Hydraulique, Tableau des forces hydrauliques de la Province de Québec, $1^{\text {er }}$ janvier 1928. 


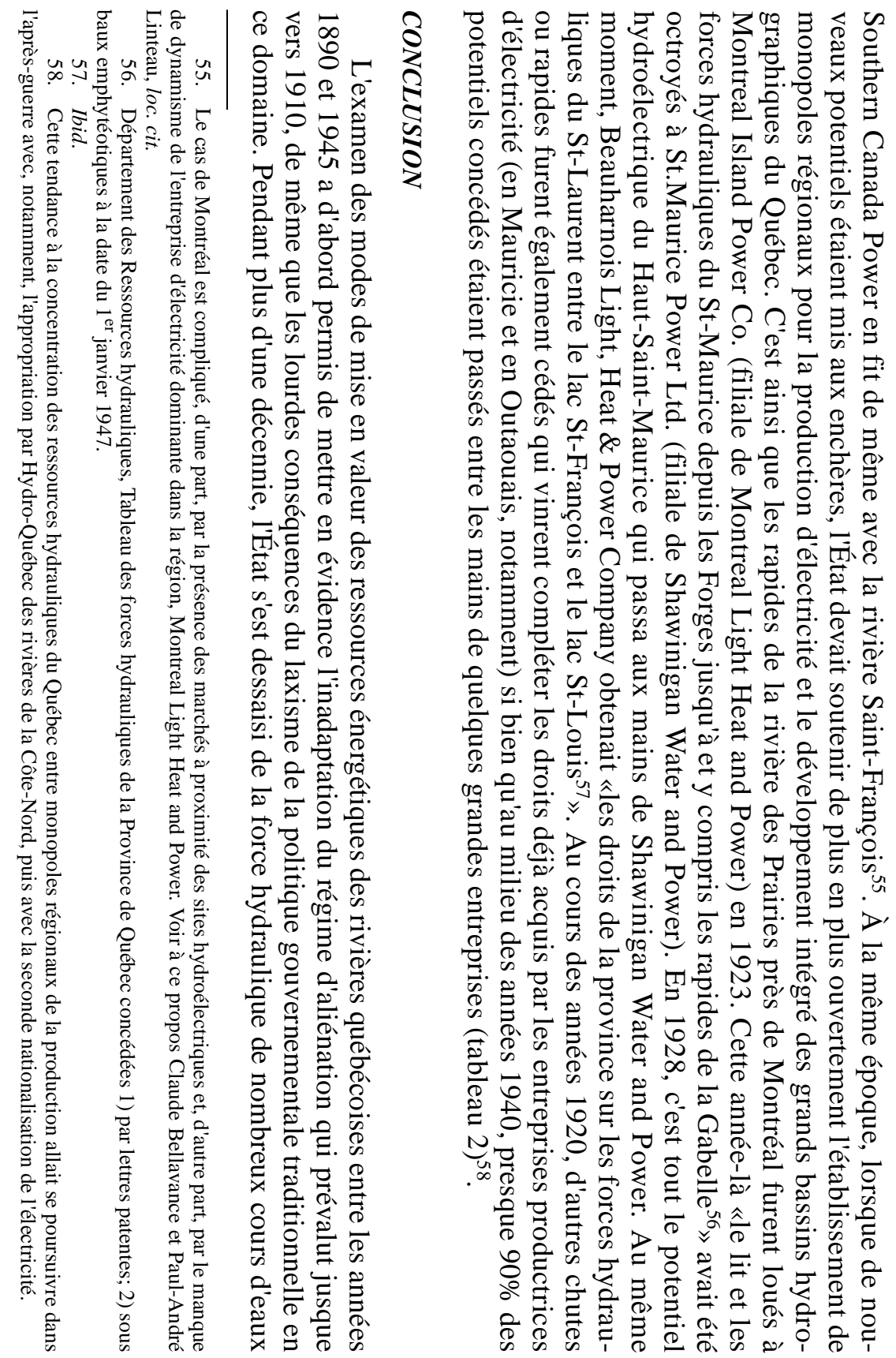




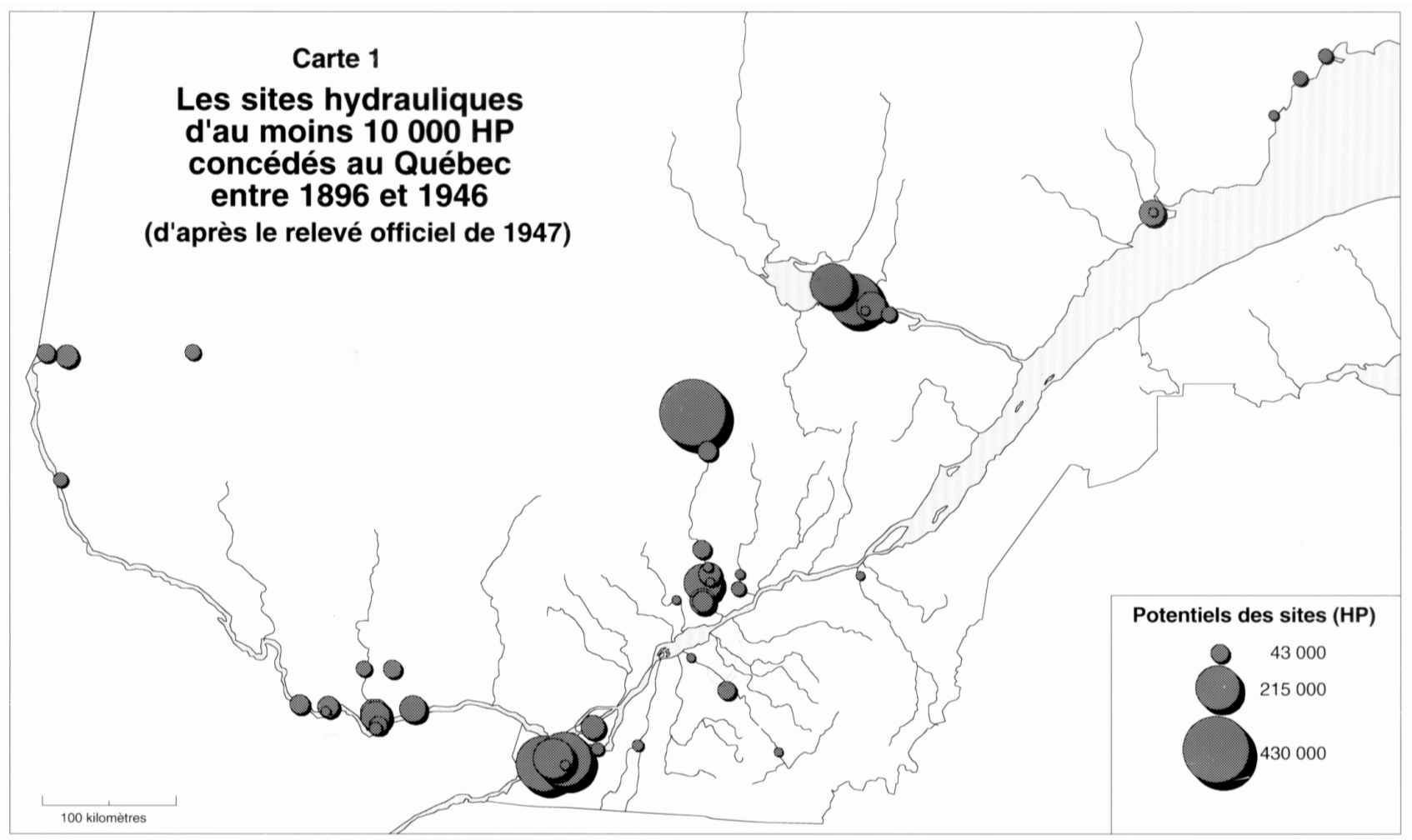

Sources: Département des Ressources hydrauliques, Tableau des forces hydrauliques de la Province de Québec concédées 1) par lettres patentes; 2) sous baux emphytéotiques à la date du 1er janvier 1947.

alors qu'il en évaluait encore très mal la valeur et qu'il n'était pas suffisamment informé de ces propriétés particulières. À cette époque, les potentiels étaient vendus en pleine propriété à des entrepreneurs de tous ordres, les spéculateurs se disputant les droits d'exploitation avec les petits industriels et les dirigeants d'entreprises d'électricité locales. La première génération d'emplacements cédés répondait essentiellement aux besoins encore limités des entreprises industrielles et l'on se souciait peu alors de voir se multiplier les établissements le long d'un cours d'eau.

Les transformations rapides des techniques de production et de transport, de pair avec le développement des marchés de l'électricité, conduisirent à l'élaboration de projets d'aménagement de plus en plus ambitieux. 
TABLEAU 2

\section{LE PARTAGE DES SITES OFFRANT UN POTENTIEL SUPÉRIEUR À 10000 HP}

\begin{tabular}{lrrl} 
& \multicolumn{1}{c}{ HP } & \multicolumn{1}{c}{$\%$} & Principal cours d'eau \\
Groupe Shawinigan & 957850 & 25,7 & Saint-Maurice \\
Hydro-Québec & 939600 & 25,2 & Saint-Laurent (section Beauharnois) \\
& & & \\
Alcan/Saguenay Power & 570000 & 15,3 & Saguenay \\
Gatineau Power & 423000 & 11,3 & Outaouais et Gatineau \\
MacLaren-Quebec & 385000 & 10,3 & Lièvre \\
Power & & & \\
Southern Canada Power & 53400 & 2,4 & Saint-François \\
Autres & 398180 & 10,7 & \\
Total & 3727030 & 100,0 &
\end{tabular}

Remarque: N'ont pas été considérées ici les sections de la rivière Outaouais cédées à l'Ontario en vertu d'une entente sur le partage de son potentiel (puissance évaluée à 345100 HP).

Source: Département des Ressources hydrauliques, Tableau des forces hydrauliques de la Province de Québec concédées 1) par lettres patentes; 2) sous baux emphytéotiques à la date du $1^{\mathrm{er}}$ janvier 1947.

Une réforme du mode de gestion de la ressource allait bientôt s'imposer. La taille des barrages projetés devenait telle, en effet, qu'on ne pouvait plus négliger leur impact sur les installations situées sur le même cours. Qui plus est, les ingénieurs hydrauliciens conçurent, à partir des années 1910, des dispositifs permettant de régulariser le débit des rivières et d'augmenter sensiblement la puissance qu'on pouvait en tirer. Le barrage Gouin, à l'origine de la formation, en 1917, d'un immense réservoir à la tête de la rivière Saint-Maurice, constitue le premier de ces ouvrages d'envergure dont l'impact se fait toujours sentir.

Ces réalisations laissaient entrevoir tous les avantages d'une gestion unifiée des grands bassins hydrographiques du Québec méridional. Aussi l'État fut-il amené à reconsidérer le mode de mise en valeur de la ressource jusque-là privilégié. La première mesure adoptée en ce sens fut de remplacer la vente en propriété par le bail emphytéotique; la seconde, de procéder à une évaluation rigoureuse des potentiels des rivières ainsi qu'à leur classement. Mais ces mesures n'étaient pas suffisantes pour répondre aux nouveaux impératifs de production. Seul l'État pouvait, au nom de l'intérêt public, limiter les droits des riverains, forcer les détenteurs de 
pouvoirs d'eau à gérer l'ouverture des vannes en fonction d'un plan d'ensemble et leur faire assumer une partie des frais encourus.

La Commission des eaux courantes, l'organisme gouvernemental qui avait été chargé de la reconnaissance des potentiels des cours d'eau, fut le maître d'œuvre des premiers ouvrages de régulation au Québec. À partir des années 1920 cependant, son intervention allait être moins directe. Au Saguenay, par exemple, c'est Alcoa qui imposa ses conditions: elle procéda elle-même à l'érection des barrages et voulut contrôler le débit de la rivière à la sortie du lac Saint-Jean. Si des crues exceptionnelles et la résistance de la population locale forcèrent la firme américaine à certains compromis, le recul de l'État n'en était pas moins évident. Aussi, bien que d'autres réservoirs allaient être créés à la fin des années 1920 et au début de la décennie suivante (aux sources de la Gatineau et de la Lièvre, notamment), le rôle de la Commission allait maintenant s'avérer plutôt marginal.

L'autre aspect central de l'histoire de l'hydroélectricité à cette époque fut l'appui non équivoque de l'État à la formation de monopoles régionaux de production d'électricité. Plusieurs des entreprises concernées figuraient parmi les plus grandes sociétés à l'échelle canadienne. Elles étaient d'ores et déjà suffisamment puissantes pour racheter - souvent au gros prix - les chutes qui leur échappaient ou encore pour s'imposer lors de la mise aux enchères de nouveaux sites. La montée en force des grands réseaux régionaux au cours des années 1920 a donc favorisé la rationalisation des conditions d'exploitation des potentiels, tout en consacrant l'appropriation de la ressource par des intérêts privés de même que le caractère désormais hautement monopoliste de l'industrie de l'électricité. Un examen de l'évolution de l'ensemble du secteur industriel montrerait que ce sont les mêmes sociétés qui, tout en s'appropriant les bassins hydrographiques à fort potentiel, procédaient au partage des marchés et au découpage du territoire en quelques grandes zones d'influence ${ }^{59}$.

Sans pour autant prétendre avoir levé le voile sur les diverses facettes de l'affirmation du capitalisme de grande organisation et le recul des formes traditionnelles de la concurrence, cette étude aura tout de même indiqué, d'une part, dans quel contexte l'État fut amené à assumer des rôles inédits dans le champ économique et, d'autre part, comment les régulations étatiques, loin de se substituer aux nouvelles régulations marchandes, vinrent au contraire les appuyer ou les consolider durant les premières décennies du siècle.

59. Claude Bellavance, «Réseaux, territoires et électricité», loc. cit. 